\title{
Kramers degeneracy in a magnetic field and Zeeman spin-orbit coupling in antiferromagnetic conductors
}

\author{
Revaz Ramazashvili: \\ ENS, LPTMS, UMR8626, Bât. 100, Université Paris-Sud, 91405 Orsay, France
}

(Dated: October 23, 2018)

\begin{abstract}
In this article, I analyze the symmetries and degeneracies of electron eigenstates in a commensurate collinear antiferromagnet. In a magnetic field transverse to the staggered magnetization, a hidden anti-unitary symmetry protects double degeneracy of the Bloch eigenstates at a special set of momenta. In addition to this 'Kramers degeneracy' subset, the manifold of momenta, labeling the doubly degenerate Bloch states in the Brillouin zone, may also contain an 'accidental degeneracy' subset, that is not protected by symmetry and that may change its shape under perturbation. These degeneracies give rise to a substantial momentum dependence of the transverse $g$-factor in the Zeeman coupling, turning the latter into a spin-orbit interaction.

I discuss a number of materials, where Zeeman spin-orbit coupling is likely to be present, and outline the simplest properties and experimental consequences of this interaction, that may be relevant to systems from chromium to borocarbides, cuprates, hexaborides, iron pnictides, as well as organic and heavy fermion conductors.
\end{abstract}

PACS numbers: $75.50 . \mathrm{Ee}$

\section{INTRODUCTION}

Antiferromagnetism is widespread in materials with interesting electron properties. Chromium [1] and its alloys [2, 3], numerous borocarbides [4], electron- and holedoped cuprates [5, 6], iron pnictides 7], various organic [8] and heavy fermion [9, 10, 11] compounds all have an antiferromagnetic state present in their phase diagram. The physics of these antiferromagnetic phases has been a subject of active research.

In this article, I study the response of electron Bloch eigenstates in an antiferromagnet to a weak magnetic field. I concentrate on the simplest case: a centrosymmetric doubly commensurate collinear antiferromagnet, shown schematically in Fig. 11, where the magnetization density at any point in space is parallel or anti-parallel to a single fixed direction $\mathbf{n}$ of the staggered magnetization, and changes sign upon primitive translation of the underlying lattice.

In a paramagnet, the double degeneracy of the Bloch eigenstates is commonly attributed to symmetry under time reversal $\theta$ - and, indeed, perturbations that break time reversal symmetry (such as ferromagnetism or a magnetic field) do tend to remove the degeneracy. Yet violation of $\theta$ alone does not preclude degeneracy: in a commensurate centrosymmetric Néel antiferromagnet, as in a paramagnet, all Bloch eigenstates enjoy a Kramers degeneracy [12] in spite of time reversal symmetry being broken in the former, but not in the latter.

In an antiferromagnet, the staggered magnetization sets a special direction $\mathbf{n}$ in electron spin space, making it anisotropic. A magnetic field along $\mathbf{n}$ removes the degeneracy of all Bloch eigenstates, as it does in a paramagnet. By contrast, in a transverse field, a hidden anti-unitary symmetry protects the Kramers degeneracy of Bloch eigenstates at a special set of momenta.

Generally, in $d$ dimensions, the manifold of momenta, corresponding to doubly degenerate Bloch states in a transverse field is $(d-1)$-dimensional; within a subset of this manifold, the degeneracy is dictated by symmetry. This is in marked contrast with what happens in a paramagnet, where an arbitrary magnetic field lifts the degeneracy of all Bloch eigenstates. For brevity, in this article I often refer to the manifold of momenta, labeling the degenerate Bloch states in the Brillouin zone, as to the 'degeneracy manifold'.

As a consequence of the Kramers degeneracy of the special Bloch states in a transverse field, the transverse component $g_{\perp}$ of the electron $g$-tensor vanishes for such states. Not being identically equal to zero, $g_{\perp}$ must, therefore, carry a substantial momentum dependence, and the Zeeman coupling $\mathcal{H}_{Z S O}$ must take the form

$$
\mathcal{H}_{Z S O}=-\mu_{B}\left[g_{\|}\left(\mathbf{H}_{\|} \cdot \boldsymbol{\sigma}\right)+g_{\perp}(\mathbf{p})\left(\mathbf{H}_{\perp} \cdot \boldsymbol{\sigma}\right)\right],
$$

where $\mathbf{H}_{\|}=(\mathbf{H} \cdot \mathbf{n}) \mathbf{n}$ and $\mathbf{H}_{\perp}=\mathbf{H}-\mathbf{H}_{\|}$are the longitudinal and transverse components of the magnetic field with respect to the unit vector $\mathbf{n}$ of the staggered magnetization, $\mu_{B}$ is the Bohr magneton, while $g_{\|}$and $g_{\perp}(\mathbf{p})$ are the longitudinal and transverse components of the $g$-tensor.

This significant momentum dependence of $g_{\perp}(\mathbf{p})$ turns the common Zeeman coupling into a kind of spin-orbit interaction $\mathcal{H}_{Z S O}$ (11), whose appearance and key properties are the focus of this work. Zeeman spin-orbit coupling may manifest itself spectacularly in a number of ways, which will be mentioned below and discussed in detail elsewhere.

The symmetry properties of wave functions in magnetic crystals have been studied by Dimmock and Wheeler [13], who pointed out, among other things, that 
magnetism not only lifts degeneracies by obviously lowering the symmetry, but also may introduce new ones. This may happen at the magnetic Brillouin zone (MBZ) boundary, under the necessary condition that the magnetic unit cell be larger than the paramagnetic one [13].

For a Néel antiferromagnet on a lattice of square symmetry, the response of the electron states to a magnetic field was studied in Ref. 14] using symmetry arguments, and in Ref. [15] within a weak coupling model. The present work is a detailed presentation of recent results [16]. It revisits Ref. [14], extends it to an arbitrary crystal symmetry and to a finite as opposed to infinitesimal magnetic field, and uncovers a rich interplay between the symmetry of magnetic structure and that of the underlying crystal lattice. At the same time, the present work extends Ref. [13] by allowing for an external magnetic field - to show how, at special momenta, the Kramers degeneracy in an antiferromagnet may persist even in a transverse magnetic field.

This work treats antiferromagnetic order as static, neglecting both its classical and quantum fluctuations. This excludes from consideration strongly fluctuating antiferromagnetic states such as those near a continuous phase transition, be it a finite-temperature Néel transition or a quantum $(T=0)$ critical point. At the same time, the single-electron Bloch eigenstates, considered hereafter, must be well-defined. As in a normal Fermi liquid state, this does not rule out strong interaction between electrons, but simply requires temperatures well below the Fermi energy. Finally, to justify the neglect of quantum fluctuations, the ordered magnetic moment must be of the order of or greater than the Bohr magneton.

As a consequence, the present theory applies to antiferromagnets (i) deep inside a commensurate long-range antiferromagnetic state, and far enough from any continuous Néel transition, finite-temperature or quantum, (ii) with an ordered moment noticeable on the scale of the Bohr magneton, and (iii) far below both the Néel and the effective Fermi temperatures. All materials mentioned in Section IV are meant to be considered under these conditions.

The article is organized as follows. Section II opens with a reminder of how, in spite of broken time reversal symmetry, all Bloch eigenstates in a commensurate collinear antiferromagnet retain Kramers degeneracy, provided there is an inversion center [12]. Then I show how, even in a transverse magnetic field, a hidden symmetry of antiferromagnetic order may protect the Kramers degeneracy for certain Bloch states.

Section III establishes several properties of the singleelectron spectrum in a weakly coupled antiferromagnet, subject to a transverse magnetic field.

Section IV contains the analysis of simple examples, that may be relevant to specific materials from chromium to organic conductors, from borocarbides to underdoped cuprates and to various heavy fermion metals. The Dis- cussion section reviews the findings, and examines them in the light of earlier work, while the Appendices present various technical details.

\section{GENERAL ARGUMENTS}

It is convenient to begin by describing the crystal host symmetry in the absence of magnetism, with the average magnetization density notionally set to zero [13]. I refer to this as to the paramagnetic state symmetry, even though the symmetry of the actual paramagnetic state may be different, for instance due to a lattice distortion upon transition. Unitary symmetries of the paramagnetic state form a group, $h$, which includes a set of elementary translations $\mathbf{T}_{\mathbf{a}}$ by primitive translation vectors a. Time reversal $\theta$ being indeed a symmetry of the paramagnetic state, the full symmetry group $g$ of the paramagnetic state includes $h$, and products of $\theta$ with each element of $h: g=h+\theta \cdot h$; put otherwise, $h$ is an invariant unitary subgroup of $g$.

Antiferromagnetic order couples to the electron spin $\boldsymbol{\sigma}$ via the exchange term $\left(\boldsymbol{\Delta}_{\mathbf{r}} \cdot \boldsymbol{\sigma}\right)$, where $\boldsymbol{\Delta}_{\mathbf{r}}$ is proportional to the average microscopic magnetization at point r. In keeping with the arguments of the Introduction, fluctuations of $\boldsymbol{\Delta}_{\mathrm{r}}$ are neglected. Being of relativistic origin, spin-orbit couplings of the crystal lattice to the electron spin and to the magnetization density are also neglected. This is a good approximation in a broad range of problems, at the very least at temperatures above the scale set by the spin-orbit coupling (see Section V for details). In this 'exchange symmetry' approximation [17], magnetization density and electron spin are assigned to a separate space, independent of the real space of the crystal; this makes coordinate rotations and other point symmetries inert with respect to $\Delta_{\mathrm{r}}$ and $\boldsymbol{\sigma}$.

A nonzero $\boldsymbol{\Delta}_{\mathbf{r}}$ changes sign under time reversal $\theta$, and removes the symmetry under primitive translations $\mathbf{T}_{\mathbf{a}}$, thus reducing the symmetry with respect to that of paramagnetic state. In a doubly commensurate collinear antiferromagnet, $\boldsymbol{\Delta}_{\mathbf{r}}$ changes sign upon $\mathbf{T}_{\mathrm{a}}: \boldsymbol{\Delta}_{\mathbf{r}+\mathbf{a}}=-\boldsymbol{\Delta}_{\mathbf{r}}$, while $\mathbf{T}_{\mathbf{a}}^{2}$ leaves $\Delta_{\mathbf{r}}$ intact: $\boldsymbol{\Delta}_{\mathbf{r}+2 \mathbf{a}}=\boldsymbol{\Delta}_{\mathbf{r}}$. Even though neither $\theta$ nor $\mathbf{T}_{\mathbf{a}}$ remain a symmetry, their product $\theta \mathbf{T}_{\mathbf{a}}$ does (see Fig. 1). In a system with inversion center, so does $\theta \mathbf{T}_{\mathbf{a}} \mathcal{I}$, where $\mathcal{I}$ is inversion. The importance of the combined symmetry $\theta \mathbf{T}_{\mathbf{a}} \mathcal{I}$ will become clear in the next subsection.

Together with the uniaxial character $\left(\boldsymbol{\Delta}_{\mathbf{r}}\right.$ at any point $\mathbf{r}$ pointing along or against the single direction $\mathbf{n}$ of staggered magnetization), these relations define a commensurate collinear Néel antiferromagnet via transformation properties of its microscopic magnetization density. 


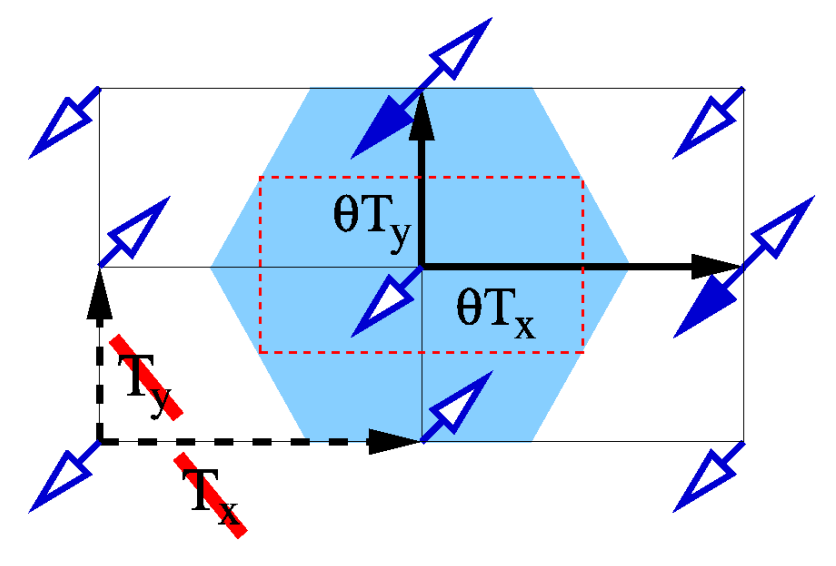

FIG. 1: (color online). Doubly commensurate collinear antiferromagnet on a simple rectangular lattice. In the absence of magnetism, time reversal $\theta$ and primitive translations $\mathbf{T}_{x}$ and $\mathbf{T}_{y}$, shown by dashed arrows, are symmetry operations. In the antiferromagnetic state, neither of the three remains a symmetry, but the products $\theta \mathbf{T}_{x}$ and $\theta \mathbf{T}_{y}$, shown by solid arrows, do, as illustrated by filled spin arrows. Small dashed rectangle at the center is the Wigner-Seitz cell boundary in the paramagnetic state, while the shaded hexagon is its antiferromagnetic counterpart. Notice that neither of the point group operations interchanges the two sublattices, hence any point symmetry of the lattice, including inversion $\mathcal{I}$, remains a symmetry of the antiferromagnetic state.

\section{Kramers degeneracy in zero field}

The combined anti-unitary symmetry $\theta \mathbf{T}_{\mathbf{a}} \mathcal{I}$ gives rise to a Kramers degeneracy [12]: If $|\mathbf{p}\rangle$ is a Bloch eigenstate at momentum $\mathbf{p}$, then $\theta \mathbf{T}_{\mathbf{a}} \mathcal{I}|\mathbf{p}\rangle$ is degenerate with $|\mathbf{p}\rangle$. Since $\theta$ and $\mathcal{I}$ both invert the momentum, both $|\mathbf{p}\rangle$ and $\theta \mathbf{T}_{\mathbf{a}} \mathcal{I}|\mathbf{p}\rangle$ carry the same momentum label p. Formally, this is verified by the action of any translation $\mathbf{T}_{\mathbf{b}}$, that remains a symmetry of the antiferromagnetic state:

$$
\begin{aligned}
& \mathbf{T}_{\mathbf{b}} \theta \mathbf{T}_{\mathbf{a}} \mathcal{I}|\mathbf{p}\rangle=\theta \mathbf{T}_{\mathbf{a}} \mathbf{T}_{\mathbf{b}} \mathcal{I}|\mathbf{p}\rangle=\theta \mathbf{T}_{\mathbf{a}} \mathcal{I} \mathbf{T}_{-\mathbf{b}}|\mathbf{p}\rangle= \\
= & \theta \mathbf{T}_{\mathbf{a}} \mathcal{I} e^{-i \mathbf{p} \cdot \mathbf{b}}|\mathbf{p}\rangle=e^{i \mathbf{p} \cdot \mathbf{b}} \theta \mathbf{T}_{\mathbf{a}} \mathcal{I}|\mathbf{p}\rangle .
\end{aligned}
$$

At the same time, $|\mathbf{p}\rangle$ and $\theta \mathbf{T}_{\mathbf{a}} \mathcal{I}|\mathbf{p}\rangle$ are orthogonal. This follows from Eqn. (22) of Appendix A as soon as one chooses $\mathcal{O}=\mathbf{T}_{\mathbf{a}} \mathcal{I},|\psi\rangle=|\mathbf{p}\rangle$, and $|\phi\rangle=\mathbf{T}_{\mathbf{a}} \mathcal{I} \theta|\mathbf{p}\rangle$. Recalling that $\left(\mathbf{T}_{\mathbf{a}} \mathcal{I}\right)^{2}=-\theta^{2}=1$, and hence $\left(\theta \mathbf{T}_{\mathbf{a}} \mathcal{I}\right)^{2}=-1$, one finds

$$
\left\langle\mathbf{p}\left|\mathcal{I} \mathbf{T}_{\mathbf{a}} \theta\right| \mathbf{p}\right\rangle=-\left\langle\mathbf{p}\left|\mathcal{I} \mathbf{T}_{\mathbf{a}} \theta\right| \mathbf{p}\right\rangle
$$

Thus, in spite of broken time reversal symmetry, in a centrosymmetric commensurate Néel antiferromagnet all Bloch states retain a Kramers degeneracy.

\section{Kramers degeneracy in a transverse field}

Generally, a magnetic field $\mathbf{H}$ lifts this degeneracy. However, in a transverse field, a hidden anti-unitary sym- metry may protect the degeneracy at a special set of points in the Brillouin zone, as I show below.

In an antiferromagnet, subject to a magnetic field $\mathbf{H}$, the single-electron Hamiltonian takes the form

$$
\mathcal{H}=\mathcal{H}_{0}+\left(\boldsymbol{\Delta}_{\mathbf{r}} \cdot \boldsymbol{\sigma}\right)-(\mathbf{H} \cdot \boldsymbol{\sigma}),
$$

where the 'paramagnetic' part $\mathcal{H}_{0}$ is invariant under independent action of $\mathbf{T}_{\mathbf{a}}$ and $\theta$, and $g \mu_{B}$ is set to unity. In the absence of the field, all Bloch eigenstates of Hamiltonian (4) are doubly degenerate by virtue of Eqn. (3).

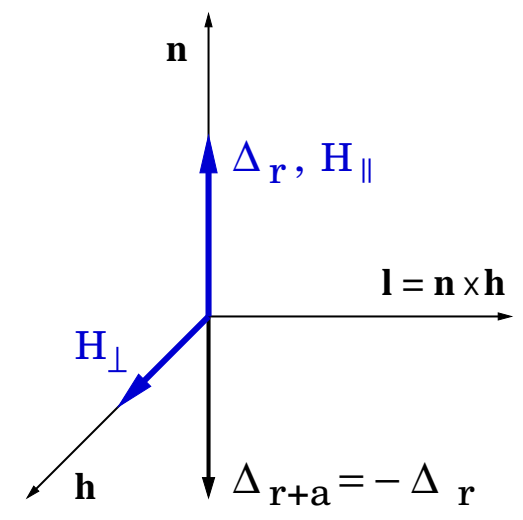

FIG. 2: (color online). Relative orientation of $\boldsymbol{\Delta}_{\mathbf{r}}, \boldsymbol{\Delta}_{\mathbf{r}+\mathbf{a}}, \mathbf{H}_{\|}$ and $\mathbf{H}_{\perp}$. To see the combined symmetries of Table \ notice that $\theta$ flips both $\boldsymbol{\Delta}_{\mathbf{r}}$ and $\mathbf{H}$, while $\mathbf{T}_{\mathbf{a}}$ leaves $\mathbf{H}$ intact, but inverts $\boldsymbol{\Delta}_{\mathbf{r}}$. Unit vectors $\mathbf{n}, \mathbf{h}$ and $\mathbf{l}=\mathbf{n} \times \mathbf{h}$ are defined as shown.

Consider the symmetries of Hamiltonian (4), involving a combination of an elementary translation $\mathbf{T}_{\mathbf{a}}$, time reversal $\theta$, or a spin rotation $\mathbf{U}_{\mathbf{m}}(\phi)$ around an axis $\mathbf{m}$ by an angle $\phi$. These symmetries are listed in Table I the relative orientation of $\boldsymbol{\Delta}_{\mathbf{r}}, \mathbf{H}_{\|}$and $\mathbf{H}_{\perp}$ is shown in Fig. 2. The transverse field $\mathbf{H}_{\perp}$ breaks the symmetries $\mathbf{U}_{\mathbf{n}}(\phi)$ and $\mathbf{T}_{\mathbf{a}} \theta$, but preserves their combination at $\phi=\pi$, i.e. $\mathbf{U}_{\mathbf{n}}(\pi) \mathbf{T}_{\mathbf{a}} \theta$. Acting on an exact Bloch state $|\mathbf{p}\rangle$ at momentum $\mathbf{p}$, this combined anti-unitary operator creates a degenerate partner eigenstate $\mathbf{U}_{\mathbf{n}}(\pi) \mathbf{T}_{\mathbf{a}} \theta|\mathbf{p}\rangle$, which is orthogonal to $|\mathbf{p}\rangle$ everywhere in the Brillouin zone, unless $(\mathbf{p} \cdot \mathbf{a})$ is an integer multiple of $\pi$ (in other words, unless p lies at a paramagnetic Brillouin zone boundary):

$$
\left\langle\mathbf{p}\left|\mathbf{U}_{\mathbf{n}}(\pi) \mathbf{T}_{\mathbf{a}} \theta\right| \mathbf{p}\right\rangle=e^{-2 i(\mathbf{p} \cdot \mathbf{a})}\left\langle\mathbf{p}\left|\mathbf{U}_{\mathbf{n}}(\pi) \mathbf{T}_{\mathbf{a}} \theta\right| \mathbf{p}\right\rangle
$$

Equation (5) follows from Eqn. (22) of Appendix A for $\mathcal{O}=\mathbf{U}_{\mathbf{n}}(\pi) \mathbf{T}_{\mathbf{a}},|\psi\rangle=|\mathbf{p}\rangle$, and $|\phi\rangle=\mathbf{U}_{\mathbf{n}}(\pi) \mathbf{T}_{\mathbf{a}} \theta|\mathbf{p}\rangle$ as soon as one observes, that $\left[\mathbf{U}_{\mathbf{n}}(\pi) \mathbf{T}_{\mathbf{a}} \theta\right]^{2}=\mathbf{T}_{\mathbf{a}}^{2}=\mathbf{T}_{2 \mathbf{a}}$. In a magnetic field, double translation $\mathbf{T}_{2 \mathbf{a}}$ remains a symmetry; according to the Bloch theorem, it acts on $|\mathbf{p}\rangle$ as per $\mathbf{T}_{2 \mathbf{a}}|\mathbf{p}\rangle=e^{2 i(\mathbf{p} \cdot \mathbf{a})}|\mathbf{p}\rangle$, thus leading to (5).

Notice, however, that the eigenstate $\mathbf{U}_{\mathbf{n}}(\pi) \mathbf{T}_{\mathbf{a}} \theta|\mathbf{p}\rangle$ carries momentum label $-\mathbf{p}$ rather than $\mathbf{p}$. By contrast with the case of zero field, combining $\mathbf{U}_{\mathbf{n}}(\pi) \mathbf{T}_{\mathbf{a}} \theta$ with inversion $\mathcal{I}$ no longer helps to produce a degenerate partner 
TABLE I: Symmetries of a collinear doubly commensurate antiferromagnet. The left column is for zero field, central column for a transverse magnetic field $\mathbf{H}_{\perp}$, and the right column for a longitudinal field $\mathbf{H}_{\|}$. As above, $\mathbf{T}_{\mathbf{a}}$ denotes elementary translation by a. As shown in Fig. 2, unit vector $\mathbf{n}$ is collinear with $\boldsymbol{\Delta}_{\mathbf{r}}$, unit vector $\mathbf{h}$ points along $\mathbf{H}_{\perp}$, and unit vector $\mathbf{l}$ is defined via $\mathbf{l}=\mathbf{n} \times \mathbf{h}$. For a general orientation of the field $\left(\mathbf{H}_{\|} \neq 0, \mathbf{H}_{\perp} \neq 0\right)$, not shown in this table, $\mathbf{U}_{\mathbf{l}}(\pi) \theta$ is the only surviving symmetry, where $\mathbf{U}_{\mathbf{m}}(\phi)$ denotes spin rotation by angle $\phi$ around the $\mathbf{m}$ axis.

\begin{tabular}{c|c|c}
\hline \hline$\left(\boldsymbol{\Delta}_{\mathbf{r}} \cdot \boldsymbol{\sigma}\right)$ & $\left(\boldsymbol{\Delta}_{\mathbf{r}} \cdot \boldsymbol{\sigma}\right)+\left(\mathbf{H}_{\perp} \cdot \boldsymbol{\sigma}\right)$ & $\left(\boldsymbol{\Delta}_{\mathbf{r}} \cdot \boldsymbol{\sigma}\right)+\left(\mathbf{H}_{\|} \cdot \boldsymbol{\sigma}\right)$ \\
\hline $\mathbf{U}_{\mathbf{n}}(\phi)$ & - & $\mathbf{U}_{\mathbf{n}}(\phi)$ \\
$\mathbf{U}_{\mathbf{h}}(\pi) \mathbf{T}_{\mathbf{a}}$ & $\mathbf{U}_{\mathbf{h}}(\pi) \mathbf{T}_{\mathbf{a}}$ & - \\
$\mathbf{U}_{\mathbf{l}}(\pi) \mathbf{T}_{\mathbf{a}}$ & - & - \\
$\mathbf{T}_{\mathbf{a}} \theta$ & $\mathbf{U}_{\mathbf{n}}(\pi) \mathbf{T}_{\mathbf{a}} \theta$ & - \\
$\mathbf{U}_{\mathbf{h}}(\pi) \theta$ & - & $\mathbf{U}_{\mathbf{h}}(\pi) \theta$ \\
$\mathbf{U}_{\mathbf{l}}(\pi) \theta$ & $\mathbf{U}_{\mathbf{l}}(\pi) \theta$ & $\mathbf{U}_{\mathbf{l}}(\pi) \theta$ \\
\hline \hline
\end{tabular}

eigenstate at the original momentum $\mathbf{p}$ : since $\theta, \mathbf{U}_{\mathbf{n}}(\pi)$, and $\mathbf{T}_{\mathbf{a}} \mathcal{I}$ all commute, and since $\left[\mathcal{I} \mathbf{U}_{\mathbf{n}}(\pi) \mathbf{T}_{\mathbf{a}} \theta\right]^{2}=1$, equation (22) of Appendix A for $\mathcal{O}=\mathcal{I} \mathbf{U}_{\mathbf{n}}(\pi) \mathbf{T}_{\mathbf{a}}$, $\psi=|\mathbf{p}\rangle$, and $\phi=\mathcal{I} \mathbf{U}_{\mathbf{n}}(\pi) \mathbf{T}_{\mathbf{a}} \theta|\mathbf{p}\rangle$ only confirms, that $\left\langle\mathbf{p}\left|\mathcal{I} \mathbf{U}_{\mathbf{n}}(\pi) \mathbf{T}_{\mathbf{a}} \theta\right| \mathbf{p}\right\rangle$ equals itself.

Thus, for an exact Bloch state $|\mathbf{p}\rangle$ at momentum $\mathbf{p}$, the anti-unitary symmetry $\mathbf{U}_{\mathbf{n}}(\pi) \mathbf{T}_{\mathbf{a}} \theta$ produces an orthogonal degenerate eigenstate $\mathbf{U}_{\mathbf{n}}(\pi) \mathbf{T}_{\mathbf{a}} \theta|\mathbf{p}\rangle$ at momentum $-\mathbf{p}$. The two momenta $\mathbf{p}$ and $-\mathbf{p}$ are different, with one key exception. It occurs for $\mathbf{p}$ at the magnetic Brillouin zone boundary, given a unitary symmetry $\mathcal{U}$, that transforms $\mathbf{-} \mathbf{p}$ into a momentum, equivalent to $\mathbf{p}$ up to a reciprocal lattice vector $\mathbf{Q}$ of the antiferromagnetic state [13]:

$$
-\mathcal{U} \mathbf{p}=\mathbf{p}+\mathbf{Q}
$$

In this case, the eigenstate $\mathcal{U} \mathbf{U}_{\mathbf{n}}(\pi) \mathbf{T}_{\mathbf{a}} \theta|\mathbf{p}\rangle$ carries momentum label $\mathbf{p}+\mathbf{Q} \equiv \mathbf{p}$, is degenerate with $|\mathbf{p}\rangle$ and orthogonal to it, thus explicitly demonstrating Kramers degeneracy at momentum $\mathbf{p}$ in a transverse field. This result is general: combined with any momentum-inverting anti-unitary symmetry, equation (6) leads to Kramers degeneracy at momentum $\mathbf{p}$ [13]. The simplest illustration, where $\mathcal{U}$ is the unity operator, is given by $\mathbf{p}=\mathbf{Q} / 2$ and shown in Figs. [5] and 6(a) for two particular cases. These and other examples are described in Section IV. Notice that, at $\mathbf{p}=\mathbf{Q} / 2$ (with $\mathcal{U}=\mathbf{1}$ ), the degeneracy in a transverse field is guaranteed even for a low crystal symmetry, provided an inversion center.

Also notice, once more, that $\mathcal{U}, \mathcal{I}$ and other point symmetries above are inert with respect to spin as a consequence of the 'exchange symmetry' approximation [17].

Hamiltonian (4) and the subsequent analysis ignored

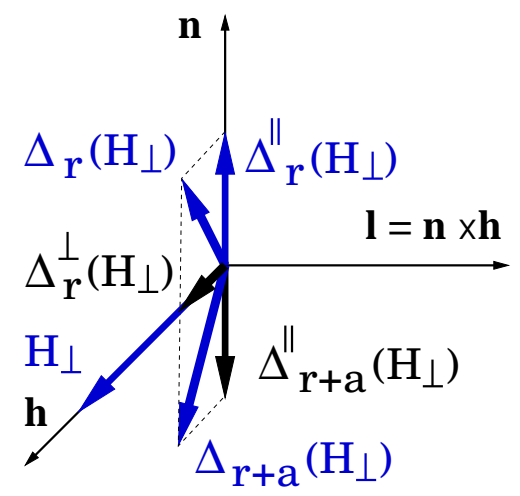

FIG. 3: (color online). Relative orientation of $\boldsymbol{\Delta}_{\mathbf{r}}\left(H_{\perp}\right)$ and $\boldsymbol{\Delta}_{\mathbf{r}+\mathbf{a}}\left(H_{\perp}\right)$, tilted by a transverse field $\mathbf{H}_{\perp}$. The component $\boldsymbol{\Delta}_{\mathbf{r}}^{\perp}\left(H_{\perp}\right)$ along the field is periodic under elementary translation $\mathbf{T}_{\mathbf{a}}: \boldsymbol{\Delta}_{\mathbf{r}+\mathbf{a}}^{\perp}\left(H_{\perp}\right)=\boldsymbol{\Delta}_{\mathbf{r}}^{\perp}\left(H_{\perp}\right)$; the component along the zero-field $\boldsymbol{\Delta}_{\mathbf{r}}$ is anti-periodic: $\boldsymbol{\Delta}_{\mathbf{r}+\mathbf{a}}^{\|}\left(H_{\perp}\right)=-\boldsymbol{\Delta}_{\mathbf{r}}^{\|}\left(H_{\perp}\right)$.

the response of the antiferromagnetic order to the transverse field $\mathbf{H}_{\perp}$. This, however, does not affect the set of points, where Kramers degeneracy in a transverse field is protected by the anti-unitary symmetry $\mathcal{U} \mathbf{U}_{\mathbf{n}}(\pi) \mathbf{T}_{\mathbf{a}} \theta$. Upon application of $\mathbf{H}_{\perp}$, the Néel sublattices tilt towards the field, making it convenient to present $\boldsymbol{\Delta}_{\mathbf{r}}$ as

$$
\boldsymbol{\Delta}_{\mathbf{r}}\left(H_{\perp}\right)=\boldsymbol{\Delta}_{\mathbf{r}}^{\perp}\left(H_{\perp}\right)+\boldsymbol{\Delta}_{\mathbf{r}}^{\|}\left(H_{\perp}\right),
$$

where $\boldsymbol{\Delta}_{\mathbf{r}}^{\perp}\left(H_{\perp}\right)$ points along $\mathbf{H}_{\perp}$, and $\boldsymbol{\Delta}_{\mathbf{r}}^{\|}\left(H_{\perp}\right)$ points along $\mathbf{n}$, as shown in Fig. 3. Since $\boldsymbol{\Delta}_{\mathbf{r}+\mathbf{a}}^{\perp}\left(H_{\perp}\right)=\boldsymbol{\Delta}_{\mathbf{r}}^{\perp}\left(H_{\perp}\right)$ and $\boldsymbol{\Delta}_{\mathbf{r}+\mathbf{a}}^{\|}\left(H_{\perp}\right)=-\boldsymbol{\Delta}_{\mathbf{r}}^{\|}\left(H_{\perp}\right)$, the second column of Table I] remains intact upon replacing $\boldsymbol{\Delta}_{\mathbf{r}}$ in Hamiltonian (4) by $\boldsymbol{\Delta}_{\mathbf{r}}\left(H_{\perp}\right)$ of Eqn. (7).

If the antiferromagnetic unit cell is a multiple of its paramagnetic counterpart, the magnetic Brillouin zone boundary contains a set of points, that do not belong to the paramagnetic Brillouin zone boundary (for example, see Figs. [5 and 6]). In the paramagnetic state, no two points of this set, separated by antiferromagnetic reciprocal lattice vector $\mathbf{Q}$ and satisfying condition (6), can be declared equivalent. As a curious consequence, the magnetic group of such a wave vector is not a subgroup of its paramagnetic counterpart [13]. Hence the degeneracy, if present, does hinge on magnetic order.

\section{CLUES FROM WEAK COUPLING}

Additional insight into the locus of states, that remain degenerate in a transverse magnetic field, is afforded by a weak-coupling single-electron Hamiltonian in a doubly commensurate collinear antiferromagnet. Let $\mathbf{Q}$ be the antiferromagnetic ordering wave vector (see the examples below); $\boldsymbol{\Delta}_{\mathbf{r}}$ creates a matrix element $(\boldsymbol{\Delta} \cdot \boldsymbol{\sigma})$ between the Bloch states at momenta $\mathbf{p}$ and $\mathbf{p}+\mathbf{Q}$ (for simplicity, I 
neglect its possible dependence on $\mathbf{p}$ ). Sublattice canting in a transverse field is taken into account in Appendix B. In magnetic field $\mathbf{H}$, and at weak coupling, Hamiltonian (41) takes the form [15]

$$
\mathcal{H}=\left[\begin{array}{cc}
\epsilon_{\mathbf{p}}-(\mathbf{H} \cdot \boldsymbol{\sigma}) & (\boldsymbol{\Delta} \cdot \boldsymbol{\sigma}) \\
(\boldsymbol{\Delta} \cdot \boldsymbol{\sigma}) & \epsilon_{\mathbf{p}+\mathbf{Q}}-(\mathbf{H} \cdot \boldsymbol{\sigma})
\end{array}\right],
$$

where $\epsilon_{\mathbf{p}}$ and $\epsilon_{\mathbf{p}+\mathbf{Q}}$ are single-particle energies of $\mathcal{H}_{0}$ in (4) at momenta $\mathbf{p}$ and $\mathbf{p}+\mathbf{Q}$, and the 'bare' $g$-tensor in $(\mathbf{H} \cdot \boldsymbol{\sigma})$ is omitted for brevity.

In a purely transverse field $\mathbf{H}_{\perp}$, this Hamiltonian can be diagonalized simply by choosing the $\hat{z}$-axis in spin space along $\mathbf{H}_{\perp}$, and the $\hat{x}$-axis along $\boldsymbol{\Delta}$. As a result, Hamiltonian (8) splits into two decoupled pieces: $\mathcal{H}_{1}\left(\mathbf{p}, \mathbf{H}_{\perp}\right)$ for the amplitudes $|\mathbf{p} ; \uparrow\rangle$ and $|\mathbf{p}+\mathbf{Q} ; \downarrow\rangle$, and $\mathcal{H}_{2}\left(\mathbf{p}, \mathbf{H}_{\perp}\right)$ for the amplitudes $|\mathbf{p} ; \downarrow\rangle$ and $|\mathbf{p}+\mathbf{Q} ; \uparrow\rangle$ :

$$
\mathcal{H}_{1(2)}\left(\mathbf{p}, \mathbf{H}_{\perp}\right)=\left[\begin{array}{cc}
\epsilon_{\mathbf{p}} \mp H_{\perp} & \Delta \\
\Delta & \epsilon_{\mathbf{p}+\mathbf{Q}} \pm H_{\perp}
\end{array}\right] .
$$

The spectra $\mathcal{E}_{1(2)}(\mathbf{p})$ of $\mathcal{H}_{1(2)}$ are given by

$$
\mathcal{E}_{1(2)}\left(\mathbf{p}, \mathbf{H}_{\perp}\right)=\eta_{\mathbf{p}} \pm \sqrt{\Delta^{2}+\left[\zeta_{\mathbf{p}} \mp\left(\mathbf{H}_{\perp} \cdot \boldsymbol{\sigma}\right)\right]^{2}},
$$

with $\left(\mathbf{H}_{\perp} \cdot \boldsymbol{\sigma}\right)=H_{\perp}$ corresponding to $\mathcal{H}_{1}$, and $\left(\mathbf{H}_{\perp} \cdot \boldsymbol{\sigma}\right)=$ $-H_{\perp}$ to $\mathcal{H}_{2}$, and with $\eta_{\mathbf{p}} \equiv \frac{\epsilon_{\mathbf{p}}+\epsilon_{\mathbf{p}+\mathbf{Q}}}{2}$, and $\zeta_{\mathbf{p}} \equiv \frac{\epsilon_{\mathbf{p}}-\epsilon_{\mathbf{p}+\mathbf{Q}}}{2}$. The same spectrum can be obtained by excluding, say, $|\mathbf{p}+\mathbf{Q} ; \sigma\rangle$ from the eigenvalue equation for (8), but it is important to keep in mind that $\boldsymbol{\sigma}$ in (10) no longer describes spin, but rather pseudospin: since $\left(\mathbf{H}_{\perp} \cdot \boldsymbol{\sigma}\right)$ does not commute with the Hamiltonian, the eigenstates of $\mathcal{H}_{1(2)}$ are superpositions of spin-up and spin-down states.

Equation (10) illustrates a number of points. Firstly, the electron spectrum acquires a gap of size $2 \Delta$. Secondly, in the absence of magnetic field, each eigenstate is indeed doubly degenerate, in agreement with the arguments, encapsulated in Eqn. (31). Thirdly, Eqn. (10) shows, that the degeneracy persists in a transverse field (and, therefore, $g_{\perp}(\mathbf{p})$ in Eqn. (1) vanishes) whenever $\zeta_{\mathbf{p}}=0$. Barring a special situation, this equation defines a surface in three dimensions, a line in two, and a set of points in one. This result for the dimensionality of the manifold of degenerate states hinges solely on the symmetry of the antiferromagnetic state and holds beyond weak coupling, as shown in Appendix C. Furthermore, as shown above, this manifold must contain all points, satisfying Eqn. (6): the points, where the degeneracy is enforced by symmetry. Finally, expansion of Eqn. (10) to first order in $\left(\mathbf{H}_{\perp} \cdot \boldsymbol{\sigma}\right)$ yields the expression for $g_{\perp}(\mathbf{p})$ in (11) within the weak coupling model (8):

$$
g_{\perp}(\mathbf{p})=\frac{\zeta_{\mathbf{p}}}{\sqrt{\Delta^{2}+\zeta_{\mathbf{p}}^{2}}} .
$$

At the end of the preceding Subsection, I showed that tilting of the Néel sublattices in a transverse field does not affect the set of points, where Kramers degeneracy in a transverse field is protected by the anti-unitary symmetry $\mathcal{U} \mathbf{U}_{\mathbf{n}}(\pi) \mathbf{T}_{\mathbf{a}} \theta$. However, generally, the rest of the degeneracy manifold is not protected by symmetry, and may change shape upon crystal deformation, or under another perturbation. For instance, while leaving intact the symmetry-protected set of degeneracy points, the sublattice canting may change the shape of the degeneracy manifold $g_{\perp}(\mathbf{p})=0$ compared with $\zeta_{\mathbf{p}}=0$. This effect is discussed in Appendix B.

Put otherwise, the degeneracy manifold may be divided into two parts. The first part is the 'Kramers degeneracy' subset of special momenta, fixed by conspiracy between the anti-unitary symmetry $\mathbf{U}_{\mathbf{n}}(\pi) \mathbf{T}_{\mathbf{a}} \theta$ and the crystal symmetry. This 'Kramers' subset is insensitive to perturbations that leave intact the crystal symmetry of the material. The rest is an 'accidental' degeneracy subset, whose geometry, by contrast, may vary under perturbations, that do not affect the crystal symmetry, but only alter the microscopic parameters of the system. The division of the degeneracy manifold into the 'Kramers' and the 'accidental' degeneracy subsets is well illustrated by the examples of two-dimensional rectangular and square symmetry antiferromagnets in Section IV.

\section{Spectral symmetries in momentum space}

The spectrum of Hamiltonian (8) enjoys a number of symmetries. Firstly, inversion symmetry makes the spectrum even under inversion. At the same time, $g_{\perp}(\mathbf{p})\left(\mathbf{H}_{\perp} \cdot \boldsymbol{\sigma}\right)$ must also be even under inversion, which implies

$$
g_{\perp}(-\mathbf{p})=g_{\perp}(\mathbf{p}) .
$$

The anti-unitary symmetry $\mathbf{U}_{\mathbf{l}}(\pi) \theta$ in the last line of Table $\prod$ is another reason for $g_{\perp}(\mathbf{p})$ to be even under inversion, as $\mathbf{U}_{\mathbf{l}}(\pi) \theta$ turns $g_{\perp}(\mathbf{p})\left(\mathbf{H}_{\perp} \cdot \boldsymbol{\sigma}\right)$ into $g_{\perp}(-\mathbf{p})\left(\mathbf{H}_{\perp} \cdot \boldsymbol{\sigma}\right)$.

Periodicity doubling due to antiferromagnetism manifests itself more interestingly. The ordering wave vector $\mathbf{Q}$ being a reciprocal lattice vector, any Bloch eigenstate at momentum $\mathbf{p}$ must have a degenerate partner eigenstate at momentum $\mathbf{p}+\mathbf{Q}$. Usually, this implies $\mathbf{Q}$-periodicity of any given band: $\epsilon(\mathbf{p})=\epsilon(\mathbf{p}+\mathbf{Q})$, which is the case, for instance, in a longitudinal field $\mathbf{H}_{\|}$, where $\mathcal{E}(\mathbf{p})$ undergoes the common Zeeman splitting $\mathcal{E}(\mathbf{p}) \rightarrow \mathcal{E}(\mathbf{p}) \pm H_{\|}$. In a Néel antiferromagnet in a transverse field, this is not the case: for a general $\mathbf{p}$, $\mathcal{E}_{1(2)}(\mathbf{p}+\mathbf{Q}) \neq \mathcal{E}_{1(2)}(\mathbf{p})$. Instead,

$$
\mathcal{E}_{1}\left(\mathbf{p}+\mathbf{Q}, \mathbf{H}_{\perp}\right)=\mathcal{E}_{2}\left(\mathbf{p}, \mathbf{H}_{\perp}\right),
$$

while both $\mathcal{E}_{1}(\mathbf{p})$ and $\mathcal{E}_{2}(\mathbf{p})$ of Eqn. (10) are invariant under momentum shift $\mathbf{p} \rightarrow \mathbf{p}+2 \mathbf{Q}$. These properties 
are illustrated in Fig. 4, showing the splitting of a onedimensional conduction band in a transverse field.

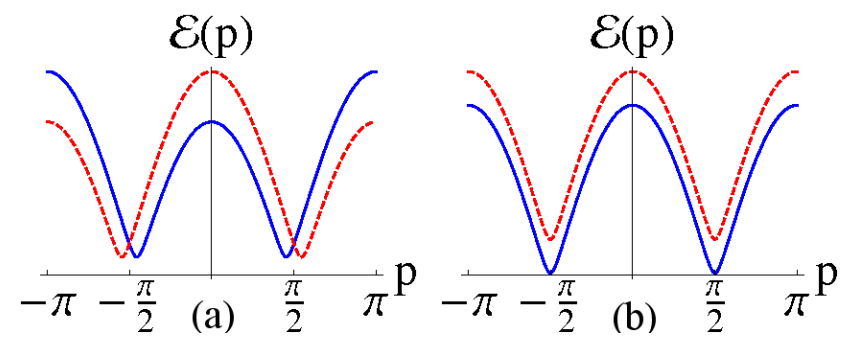

FIG. 4: (color online). One-dimensional conduction band splitting. (a) Conduction band ['+' sign in front of the square root in Eqn. (10)], split by a transverse field. Here Eqn. (13) is illustrated by spectrum (10) for a nearest-neighbor hopping $\epsilon_{\mathbf{p}}=t \cos p$ in Hamiltonian (8). Notice that, in spite of period doubling in real space, neither of the two split spectra has period $\pi$; instead, both are $2 \pi$-periodic, but map onto each other upon translation by $\pi$. Also notice that the two split sub-bands intersect at the symmetry-enforced degeneracy point $\mathbf{p}= \pm \frac{\pi}{2}$, as they should (see the one-dimensional example of the following section). (b) Conduction band, split by a longitudinal field. By contrast with (a), each split subband is $\pi$-periodic, and degeneracy is lifted for all momenta.

The reason behind (13) is that, as long as $H_{\perp} \neq 0$, neither $\mathcal{H}_{1}$ nor $\mathcal{H}_{2}$ in Eqn. (9) is invariant under the momentum boost $\mathbf{p} \rightarrow \mathbf{p}+\mathbf{Q}$, in spite of the Hamiltonians (4) and (8) both having doubled periodicity. Rather,

$$
\mathcal{H}_{1}\left(\mathbf{p}+\mathbf{Q}, \mathbf{H}_{\perp}\right)=\mathcal{H}_{2}\left(\mathbf{p}, \mathbf{H}_{\perp}\right),
$$

which is made explicit by subsequent exchange of the diagonal matrix elements, and leads to Eqn. (13).

Zeeman splitting corresponds to the difference $\mathcal{E}_{1}\left(\mathbf{p}, \mathbf{H}_{\perp}\right)-\mathcal{E}_{2}\left(\mathbf{p}, \mathbf{H}_{\perp}\right)$; hence it changes sign upon momentum shift by $\mathbf{Q}$. At the same time, direct inspection shows that Hamiltonians $\mathcal{H}_{1}$ and $\mathcal{H}_{2}$ in Eqn. (9) turn into one another upon inversion of $\mathbf{H}_{\perp}$ : $\mathcal{H}_{1}\left(\mathbf{p},-\mathbf{H}_{\perp}\right)=\mathcal{H}_{2}\left(\mathbf{p}, \mathbf{H}_{\perp}\right)$. Combining this with Eqn. (14), one finds that momentum boost by $\mathbf{Q}$ accompanied by inversion of $\mathbf{H}_{\perp}$ is a symmetry of both $\mathcal{H}_{1}$ and $\mathcal{H}_{2}$ :

$$
\mathcal{H}_{1(2)}\left(\mathbf{p}+\mathbf{Q},-\mathbf{H}_{\perp}\right)=\mathcal{H}_{1(2)}\left(\mathbf{p}, \mathbf{H}_{\perp}\right) .
$$

For the transverse Zeeman term $g_{\perp}(\mathbf{p})\left(\mathbf{H}_{\perp} \cdot \boldsymbol{\sigma}\right)$, this yields

$$
g_{\perp}(\mathbf{p}+\mathbf{Q})=-g_{\perp}(\mathbf{p}) .
$$

Combined, Eqns. (12) and (16) lead to

$$
g_{\perp}\left(\frac{\mathbf{Q}}{2}+\mathbf{p}\right)=-g_{\perp}\left(\frac{\mathbf{Q}}{2}-\mathbf{p}\right) .
$$

This implies not only that $g_{\perp}(\mathbf{p})$ must vanish at $\mathbf{p}=\frac{\mathbf{Q}}{2}$, but also that $g_{\perp}\left(\frac{\mathbf{Q}}{2}+\mathbf{p}\right)$ is an odd function of $\mathbf{p}$. The conclusions of this subsection hold after the sublattice tilting is taken into account (see Appendix B).

\section{EXAMPLES}

In this section, I describe the manifolds of degenerate states for a number of concrete examples and thus show, that the Zeeman spin-orbit coupling (1) is at work in many materials of great interest. It gives rise to various interesting phenomena, some of which are outlined in the subsection 'Experimental signatures' of Section V.

Kramers degeneracy in a transverse field and the Zeeman spin-orbit coupling (10) will manifest themselves whenever carriers are present at or near the manifold of degenerate states $g_{\perp}(\mathbf{p})=0$. In a weakly doped antiferromagnetic insulator, this will happen whenever the relevant band extremum falls at or near the manifold of degenerate states. In an antiferromagnetic metal, this occurs when the Fermi surface crosses this manifold. Hence, for metals, I mention the Fermi surface geometry whenever known.

Between these two limiting cases of a weakly doped antiferromagnetic insulator and an antiferromagnetic metal with a large Fermi surface, the experimental manifestations of the Zeeman spin-orbit coupling will be quantitatively different. On top of this, certain effects will be sensitive to the geometry of the degeneracy manifold and its intersection with the Fermi surface, as well as the orientation of the staggered magnetization with respect to the crystal axes. A detailed discussion of these effects will be presented elsewhere.

When selecting the examples below, the preference was given to materials, available in high-purity samples, where de Haas-van Alphen oscillations were observed, and where magnetic structure was unambiguously characterized by neutron scattering. As explained in the Introduction, the results of this work apply to materials well inside a long-range antiferromagnetic phase, and far enough from any critical point, quantum or classical. For both quantum and thermal fluctuations of antiferromagnetic order to be negligible, the ordered moment shall be noticeable on the scale of the Bohr magneton, and the sample shall be kept well below both the Néel and the effective Fermi temperatures.

\section{One dimension}

In one dimension, the magnetic Brillouin zone boundary reduces to two points $\mathbf{p}= \pm \frac{\pi}{2 a}$, which in fact coincide up to the antiferromagnetic wave vector $\mathbf{Q}=\frac{\pi}{a}$, that is also the reciprocal lattice vector of the antiferromagnetic state (see Fig. 5). In terms of the general condition (6), this is the simplest case: $\mathcal{U}=\mathbf{1}$.

As a result, at $\mathbf{p}= \pm \frac{\pi}{2 a}$, the two exact Bloch states in a transverse field, $|\mathbf{p}\rangle$ and $\theta \mathbf{T}_{\mathbf{a}} \mathbf{U}_{\mathbf{n}}(\pi)|\mathbf{p}\rangle$, correspond to the same momentum $\mathbf{p}$, and are degenerate by virtue of $\theta \mathbf{T}_{\mathbf{a}} \mathbf{U}_{\mathbf{n}}(\pi)$ being a symmetry. Equation (5) guarantees their orthogonality, thus protecting Kramers degeneracy 
at momentum $\mathbf{p}= \pm \frac{\pi}{2 a}$ against transverse magnetic field.

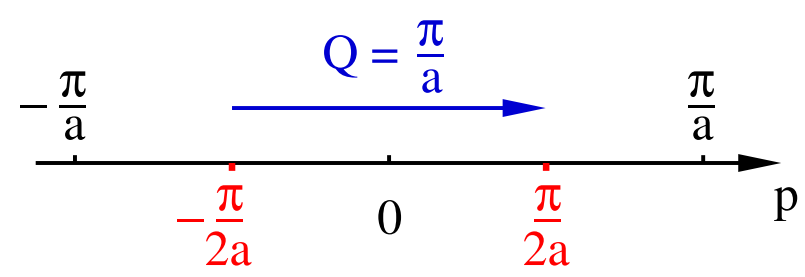

FIG. 5: (color online). The paramagnetic $\left(\mathbf{p}= \pm \frac{\pi}{a}\right)$, and the antiferromagnetic $\left(\mathbf{p}= \pm \frac{\pi}{2 a}\right)$ Brillouin zone boundaries of a one-dimensional Néel antiferromagnet. In the antiferromagnetic state, the two points $\mathbf{p}= \pm \frac{\pi}{2 a}$ are identical up to the reciprocal lattice vector $\mathbf{Q}=\frac{\pi}{a}$ of the antiferromagnetic state. At these two points, anti-unitary symmetry $\mathbf{U}_{\mathbf{n}}(\pi) \mathbf{T}_{\mathbf{a}} \theta$ protects the Kramers degeneracy against transverse magnetic field.

Two dimensions, rectangular and square symmetries

Now consider a two-dimensional antiferromagnet on a lattice of rectangular or square symmetry, with the ordering wave vector $\mathbf{Q}=(\pi, \pi)$. In a transverse magnetic field, the degeneracy persists on a line in the Brillouin zone, by virtue of Eqn. (10). I will show that, in the rectangular case, the degeneracy line must contain the point $\Sigma$ at the center of the magnetic Brillouin zone (MBZ) boundary (i.e. the star of $\mathbf{p}=\mathbf{Q} / 2$ ), shown in Fig. 6(a). In the square symmetry case, the degeneracy persists at the entire MBZ boundary (Fig. 6(b)). The MBZ in Fig. 6] is the reciprocal space counterpart of the Wigner-Seitz cell of the magnetic state (Fig. 1), and the ordering wave vector $\mathbf{Q}=(\pi, \pi)$ connects points $X$ and $Y$ in Figs. 6)(a) and (b).

Consider a Bloch state $|\mathbf{p}\rangle$ at momentum $\mathbf{p}$ in a transverse field. As discussed in Section II, the eigenstate $\theta \mathbf{T}_{\mathbf{a}} \mathbf{U}_{\mathbf{n}}(\pi)|\mathbf{p}\rangle$ at momentum $-\mathbf{p}$ is degenerate with $|\mathbf{p}\rangle$ and, according to Eqn. (5), must be orthogonal to it unless $(\mathbf{p} \cdot \mathbf{a})$ is an integer multiple of $\pi$ - put otherwise, unless $\mathbf{p}$ belongs to the paramagnetic Brillouin zone boundary. At points $\Sigma, \mathrm{X}$, and $\mathrm{Y}$, momenta $\mathbf{p}$ and $-\mathbf{p}$ coincide up to a reciprocal lattice vector of the antiferromagnetic state. However, at points $\mathrm{X}$ and $\mathrm{Y}$ (as well as at the entire vertical segment of the MBZ boundary in Fig. 6(a)), (p $\cdot \mathbf{a})$ is an integer multiple of $\pi$; hence $|\mathbf{p}\rangle$ and $\theta \mathbf{T}_{\mathbf{a}} \mathbf{U}_{\mathbf{n}}(\pi)|\mathbf{p}\rangle$ are not obliged to be orthogonal there as per Eqn. (5). Thus, $\Sigma$ is the only point at the MBZ boundary, where the two degenerate states $|\mathbf{p}\rangle$ and $\theta \mathbf{T}_{\mathbf{a}} \mathbf{U}_{\mathbf{n}}(\pi)|\mathbf{p}\rangle$ are orthogonal and correspond to the same momentum: Dashed arrows in figure 6(a) show, that, for a generic point $\mathbf{p}^{\prime}$ at the MBZ boundary, no symmetry operation relates $-\mathbf{p}^{\prime}$ to a vector, equivalent to $\mathbf{p}^{\prime}$. Therefore, it is only at point $\Sigma$, that the sym-

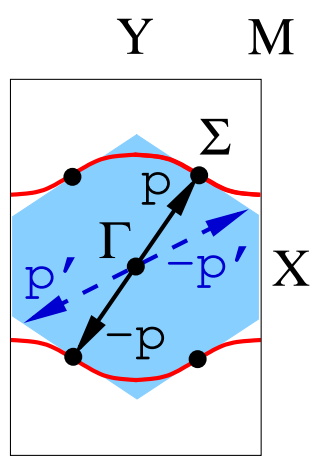

(a)

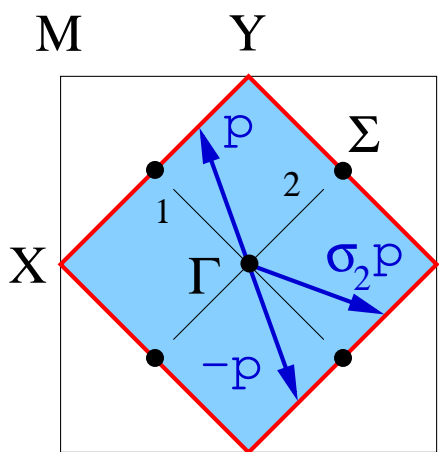

(b)
FIG. 6: (color online). Geometry of the problem. (a) The Brillouin zone for a simple rectangular lattice (the rectangle), and its antiferromagnetic counterpart (MBZ, shaded hexagon). Thick (red) curve, passing through point $\Sigma$, shows a typical degeneracy line $g_{\perp}(\mathbf{p})=0$. At the MBZ boundary, only momentum $\mathbf{p}$ at point $\Sigma$ is equivalent to $-\mathbf{p}$ up to a reciprocal lattice vector of the antiferromagnetic state; for a generic $\mathbf{p}^{\prime}$, shown by the dashed arrow, this is not true. (b) The Brillouin zone of a simple square lattice and its antiferromagnetic counterpart (shaded diagonal square). The degeneracy line must contain the entire MBZ boundary, shown in red. Point $\Gamma$ is the Brillouin zone center, points $X$ and $Y$ lie at the centers of the paramagnetic Brillouin Zone edges. Point $\Sigma$ lies at the center of the MBZ boundary.

metry protects Kramers degeneracy against transverse magnetic field. As in the one-dimensional example of the previous subsection, in terms of Eqn. (6) this corresponds to the simplest case of $\mathcal{U}=\mathbf{1}$.

This can be illustrated by a nearest-neighbor hopping spectrum

$$
\epsilon_{\mathbf{p}}=t\left[\cos p_{x}+\eta \cos p_{y}\right]
$$

in the weak-coupling example of the previous subsection: for rectangular symmetry $(\eta \neq 1)$, spectrum (10) in a transverse field remains degenerate at a thick (red) line, sketched in Fig. 6(a). Upon variation of $\eta \neq 1$, the line changes its shape, but remains pinned at the star of wave vector $\mathbf{p}=\mathbf{Q} / 2$ (i.e. at point $\Sigma$ ) in Fig. 6(a). In terms of the preceding subsection, the star of $\mathbf{p}=\mathbf{Q} / 2$ is the 'Kramers' subset of the degeneracy manifold, while the rest of the degeneracy line in Fig. 6(a) is the 'accidental' degeneracy subset.

Promotion from the rectangular symmetry $(\eta \neq 1)$ to that of a square $(\eta=1)$ brings along invariance under reflections $\sigma_{1,2}$ in either of the two diagonal axes 1 and 2, passing through point $\Gamma$ in Fig. 6(b). As a result, the eigenstate $\sigma_{1} \theta \mathbf{T}_{\mathbf{a}} \mathbf{U}_{\mathbf{n}}(\pi)|\mathbf{p}\rangle$ at momentum $\sigma_{2} \mathbf{p}$ (Fig. 6(b)) is also degenerate with $|\mathbf{p}\rangle$ and orthogonal to it, as one can show analogously to the examples above. In terms of the general condition ([6]), this means $\mathcal{U}=\sigma_{1,2}$. 
A momentum $\mathbf{p}$ at the MBZ boundary in Fig. 6(b) differs from $\sigma_{2} \mathbf{p}$ by a reciprocal lattice vector; thus the two momenta coincide in the nomenclature of the antiferromagnetic Brillouin zone. Hence, for a squaresymmetry lattice in a transverse field, the degeneracy is of a 'Kramers' (i.e. symmetry-protected) nature at the entire MBZ boundary, as shown in Fig. 6(b). In this case, barring a particularly pathological band structure, the degeneracy manifold is exhausted by its 'Kramers' subset.

In accordance with the symmetry arguments above, for the toy nearest-neighbor hopping spectrum (18) at the square symmetry point $\eta=1$, the degeneracy line of Eqn. (10) coincides with the MBZ boundary, as shown in Fig. 6(b). By contrast, for rectangular symmetry, it is Eqn. (10) that restricts the degeneracy in a transverse field to a line in momentum space, and it is the symmetry that pins this line at point $\Sigma$ at the middle of the MBZ boundary, as shown in Fig. 6(a).

Now, $g_{\perp}(\mathbf{p})$ can be expanded in a vicinity of the degeneracy line $g_{\perp}(\mathbf{p})=0$. With the exception of highersymmetry points, such as point $X$ in Fig. 6(b), the leading term of the expansion is linear in momentum deviation $\delta \mathbf{p}$ from the degeneracy line:

$$
g_{\perp}(\mathbf{p}) \approx \frac{\boldsymbol{\Xi}_{\mathbf{p}} \cdot \delta \mathbf{p}}{\hbar},
$$

where $\boldsymbol{\Xi}_{\mathbf{p}} / \hbar$ is the momentum gradient of $g_{\perp}(\mathbf{p})$ at point $\mathbf{p}$ on the degeneracy line. As mentioned in the previous Section, inversion symmetry makes $g_{\perp}(\mathbf{p})$ even under inversion. Therefore, $\boldsymbol{\Xi}_{\mathbf{p}}$ changes sign upon inversion, which is consistent with Eqns. (13) and (16), that require $g_{\perp}(\mathbf{p})$ to change sign upon momentum shift by $\mathbf{Q}$.

As shown in the last subsection of Section III, $g_{\perp}(\mathbf{p})$ is an odd function of the deviation $\delta \mathbf{p}$ from point $\Sigma$ (the star of $\mathbf{p}=\mathbf{Q} / 2$ ) in Figs. 6(a) and (b). Therefore, expansion of $g_{\perp}(\mathbf{p})$ around point $\Sigma$ cannot contain an even power of $\delta \mathbf{p}$.

\section{Chromium}

This subsection is devoted to commensurate antiferromagnetism in chromium - the simplest of magnetic orders, occurring in this textbook spin density wave metal. Chromium crystallizes into a b.c.c. lattice, and undergoes various magnetic and structural transitions upon variation of temperature, pressure, or alloying [1, 2, 3].

Below the Néel temperature $T_{N}$ of about $311 \mathrm{~K}$ at ambient pressure, chromium develops weakly incommensurate antiferromagnetism with ordered moment of about $0.5 \mu_{B}$ per atom at $4.2 \mathrm{~K}$. However, strain - or doping with some 0.1 to $0.3 \%$ of a transition metal (such as Mn, $\mathrm{Re}, \mathrm{Rh}, \mathrm{Ru}, \mathrm{Ir}, \mathrm{Os}$ or Pt [2]) - eliminate incommensurability in favor of commensurate order with wave vector [001], shown in Fig. 7. Commensurate order has

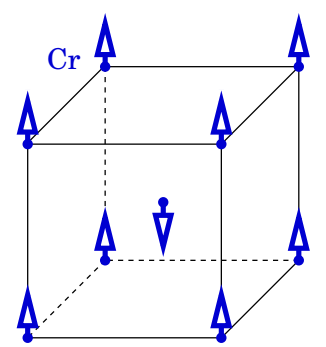

FIG. 7: (color online). Schematic drawing of the commensurate magnetic structure of antiferromagnetic chromium.
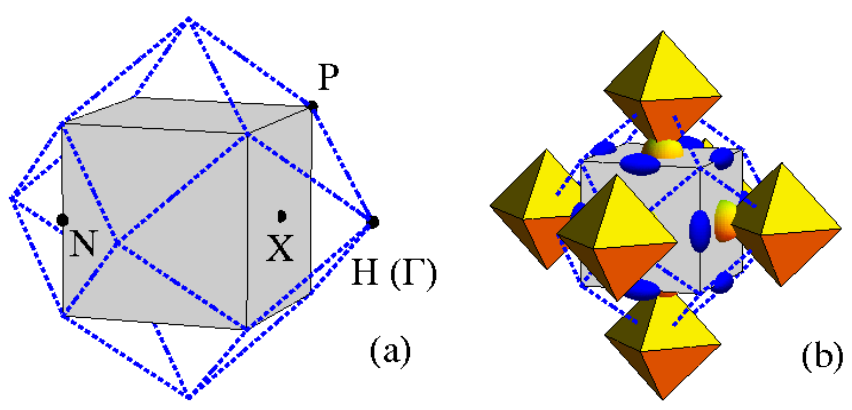

FIG. 8: (color online). The Brillouin zone and the Fermi surface of commensurate antiferromagnetic chromium. (a) Dashed lines show the paramagnetic Brillouin zone boundary. The solid cube inside it is the magnetic Brillouin zone (MBZ), with high symmetry points indicated. In the antiferromagnetic state, point $H$ is equivalent to point $\Gamma$ at the center of the Brillouin zone (not shown). (b) The Fermi surface sketch of paramagnetic chromium. As in (a), dashed lines show the paramagnetic Brillouin zone boundary, and the solid cube inside it is the MBZ. The nearly octahedral hole Fermi surface is centered at point $H$, and nearly spherical electron 'balls' are located at face centers $X$ of the MBZ boundary. Together with the nearly octahedral electron surface, centered at point $\Gamma$ (not shown), these electron balls form the electron 'jack'. A set of hole ellipsoids is centered at points $N$ in the middle of the magnetic Brillouin zone edges.

also been observed and much studied in thin films of chromium, often with an enhanced Néel temperature and ordered moment [18]. This article neglects fluctuations of magnetic order and, conveniently, the high Néel temperature of chromium facilitates experimental access to $T \ll T_{N}$, where thermal fluctuations are suppressed.

The paramagnetic and the antiferromagnetic Brillouin zones for bulk commensurate antiferromagnetic chromium are shown in Fig. 8(a). An arbitrary momentum at the MBZ boundary becomes equivalent to its opposite upon reflection in a properly chosen plane. Similarly to the two-dimensional square-symmetry example above, this equivalence is up to a primitive wave vector of the antiferromagnetic reciprocal space. Hence, in 
TABLE II: Simple properties of some of the studied samples of $\mathrm{CeIn}_{3}, \mathrm{UGa}_{3}$ and $\mathrm{UIn}_{3}$ : the Néel temperature $T_{N}$, the ordered magnetic moment $M$, the Sommerfeld coefficient $\gamma$, the residual resistivity $\rho_{0}$, and the residual resistivity ratio $\rho(300 K) / \rho_{0}$.

\begin{tabular}{c|c|c|c|c|c}
\hline \hline Material & $T_{N}$ & $\mathrm{M}$ & $\gamma$ & $\rho_{0}$ & $\frac{\rho(300 \mathrm{~K})}{\rho_{0}}$ \\
& $(\mathrm{~K})$ & $\left(\mu_{B}\right)$ & $\left(\mathrm{mJ} / \mathrm{K}^{2} \cdot \mathrm{mol}\right)$ & $(\mu \Omega \mathrm{cm})$ & \\
\hline $\mathrm{CeIn}_{3}$ & 10.1 & $\begin{array}{c}0.5 / \mathrm{Ce} \\
{[19]}\end{array}$ & 130 & $0.5[20]$ & $35[20]$ \\
& & {$[20]$} & $0.6[21]$ & $100[22]$ \\
$\mathrm{UGa}_{3}$ & 64 & $\begin{array}{c}0.75 / \mathrm{U} \\
{[23]}\end{array}$ & 52 & $1.2[26]$ & $38[25]$ \\
& {$[23]$} & $25,26]$ & & $81[26]$ \\
$\mathrm{UIn}_{3}$ & 88 & $1 / \mathrm{U}[27]$ & $50[24]$ & $0.66[28]$ & $130[28]$ \\
\hline \hline
\end{tabular}

a transverse magnetic field, the Kramers degeneracy survives at the entire magnetic Brillouin zone boundary in Fig. 8(a).

The disappearance of $g_{\perp}(\mathbf{p})$ affects electrons at two different sheets of the Fermi surface, sketched in Fig. 8(b): those at the nearly spherical electron parts, centered at points $X$ in the middle of each MBZ face, and those at the hole ellipsoids, centered at points $N$ in the middle of each MBZ edge. For the former, the leading term of the expansion is linear in the momentum deviation $\delta p_{\perp}$ from the flat face of the MBZ boundary. For the latter, the leading term of the expansion is quadratic near each MBZ edge, since $g_{\perp}(\mathbf{p})$ vanishes at each of the two intersecting faces of the MBZ boundary.

\section{$\mathrm{CeIn}_{3}, \mathrm{UIn}_{3}, \mathrm{UGa}_{3} \ldots$}

A number of cerium and uranium binary intermetallics of simple cubic $\mathrm{Cu}_{3} \mathrm{Au}$ structure, such as $\mathrm{CeIn}_{3}, \mathrm{CeTl}_{3}$, $\mathrm{UIn}_{3}, \mathrm{UGa}_{3}, \mathrm{UTl}_{3}$ and $\mathrm{UPb}_{3}$, turn antiferromagnetic at low temperatures. High-purity samples of $\mathrm{CeIn}_{3}, \mathrm{UIn}_{3}$, and $\mathrm{UGa}_{3}$ have made it possible to characterize magnetic order and electron properties of these materials rather comprehensively. Some of the basic properties of the samples are shown in Table II.

At low temperature, all three develop a type-II antiferromagnetic structure with wave vector $\mathbf{Q}=\left[\frac{1}{2} \frac{1}{2} \frac{1}{2}\right]$, shown in Fig. 9(a) for $\mathrm{CeIn}_{3}$. The materials remain normal metals down to the lowest temperatures probed, with the Sommerfeld coefficient substantially enhanced by comparison with that of a simple metal (see the fourth column of Table $\Pi$ versus about $0.65 \mathrm{~mJ} / \mathrm{K}^{2} \cdot \mathrm{mol}$ for $\mathrm{Ag}$ ).

Of the three materials, $\mathrm{CeIn}_{3}$ has been scrutinized the most. Its early studies were driven by interest in valence
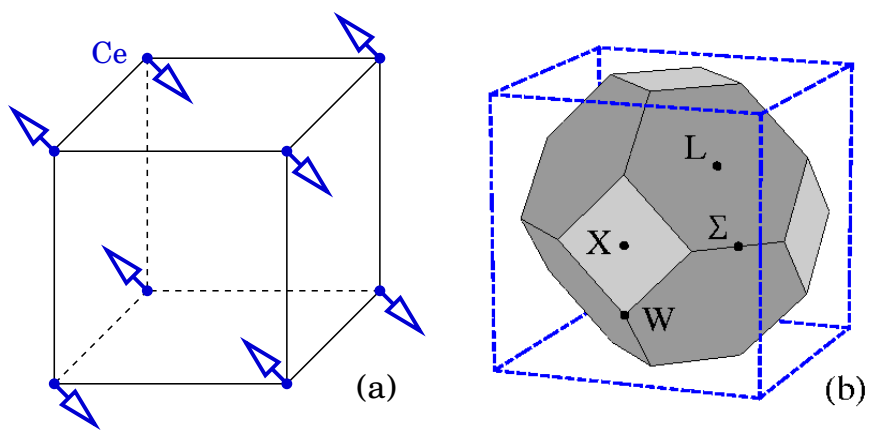

FIG. 9: (color online). Geometry of $\mathrm{CeIn}_{3}$ in real and in reciprocal space. (a) Cubic unit cell of $\mathrm{CeIn}_{3}$, showing Ce atoms and their magnetic moments. Indium atoms (not shown) are positioned at the face centers of the unit cell. (b) Cubic Brillouin zone of paramagnetic $\mathrm{CeIn}_{3}$ and, inside, its antiferromagnetic counterpart. Darker shading marks the degeneracy surface.

29] and magnetic [19] fluctuations, in the nature of its magnetic order [19], in large mass enhancement [30] and related questions. Subsequent research focused on the reduction of $T_{N}$ under pressure, and on superconductivity, discovered near the critical pressure $p_{c}$, where the Néel temperature is about to vanish - as well as on marked departure from Landau Fermi liquid behavior, found in the normal state near $p_{c}$ 222, 31, 32]. The most recent work included de Haas-van Alphen oscillation measurements [21, 33], electron-positron annihilation experiments [34], and interpretation of the former [35].

According to Fig. 9(b), the Magnetic Brillouin Zone of the three metals enjoys full cubic symmetry. Its square faces belong to the paramagnetic Brillouin zone boundary $(\mathbf{p} \cdot \mathbf{a})= \pm \pi$, where Eqn. (5) does not enforce degeneracy; however, $g_{\perp}(\mathbf{p})$ does vanish at the hexagonal MBZ faces, marked by darker shading in Fig. 9(b).

According to de Haas-van Alphen measurements 21, 36] and to calculations [37], one sheet of the Fermi surface of $\mathrm{CeIn}_{3}$ is nearly spherical, and has radius of about $\frac{\pi}{a} \frac{\sqrt{3}}{2}$, where $a$ is the lattice constant. Hence this sheet comes close to the point L in Fig. 9(b), which is the very same distance $\frac{\pi}{a} \frac{\sqrt{3}}{2}$ away from the Brillouin zone center. Disappearance of $g_{\perp}(\mathbf{p})$ necessarily affects the dynamics of an electron on this sheet in a transverse field.

Near a generic point at an MBZ face, far from its edges, leading terms of the expansion of $g_{\perp}(\mathbf{p})$ are linear in transverse deviation of momentum from the MBZ face as per Eqn. (19), with $\boldsymbol{\Xi}_{\mathbf{p}}$ normal to the MBZ boundary. Near the edges, joining the neighboring hexagonal faces in Fig. 9(b) - for instance, near the points $\Sigma$ and $W$ the leading terms become quadratic. 


\section{Uranium nitride}

Uranium nitride (UN) presents another example of interest. This heavy fermion metal has a face-centered cubic lattice of $\mathrm{NaCl}$ type, shown in Fig. 10(a). Below 53K, it develops type-I antiferromagnetic order, with ordered moment of about $0.75 \mu_{B}$ per uranium atom [38], and the Sommerfeld coefficient of $50 \mathrm{~mJ} / \mathrm{K}^{2} \cdot \mathrm{mol}[39]$. The Neéel temperature of UN drops under pressure, vanishing at about 3.5 GPa. Recent experiments [39] studied the lowtemperature resistivity near the critical pressure on samples with residual resistivity $\rho_{0}$ of about $2.3 \mu \Omega \mathrm{cm}$, and the residual resistivity ratio $\rho(300 K) / \rho_{0}$ of the order of $10^{2}$.
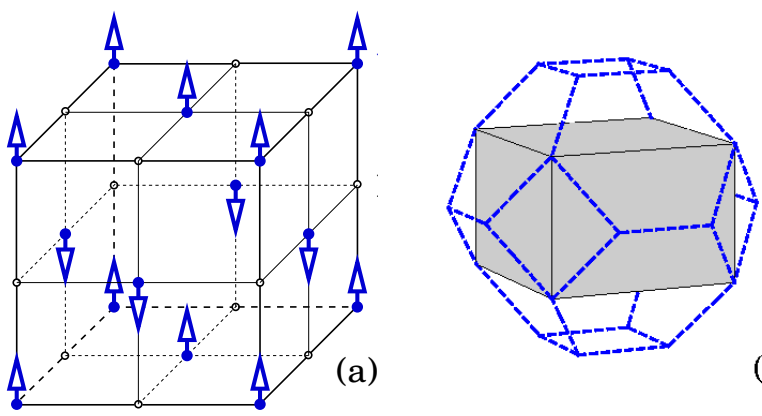

(b)

FIG. 10: (color online). Geometry of uranium nitride (UN) in real and in reciprocal space. (a) F.c.c. cubic unit cell of $\mathrm{UN}$, showing $\mathrm{U}$ atoms and their magnetic moment orientation. Nitrogen atoms are shown by open circles. (b) Dashed lines define the Brillouin zone boundary of paramagnetic UN; the square prism inside it is the antiferromagnetic Brillouin zone. Its entire boundary defines the degeneracy surface $g_{\perp}(\mathbf{p})=0$.

The real-space sketch of magnetic structure of UN is shown in Fig. 10 together with its paramagnetic and antiferromagnetic Brillouin zone boundaries. The MBZ has full tetragonal symmetry and, in a transverse field, all the states at its boundary retain Kramers degeneracy. The leading terms in the expansion of $g_{\perp}(\mathbf{p})$ are linear near the MBZ faces, quadratic near the edges, and cubic near the vertices.

\section{$\mathrm{CePd}_{2} \mathrm{Si}_{2}$ and $\mathrm{CeRh}_{2} \mathrm{Si}_{2}$}

The heavy fermion metal $\mathrm{CePd}_{2} \mathrm{Si}_{2}$ has a bodycentered tetragonal structure of $\mathrm{ThCr}_{2} \mathrm{Si}_{2}$ type, shown in Fig. 11(a). It is isostructural to $\mathrm{CeCu}_{2} \mathrm{Si}_{2}-$ the first discovered heavy fermion superconductor [40] and $\mathrm{CeCu}_{2} \mathrm{Ge}_{2}$, an incommensurate antiferromagnet [41], that becomes superconducting above $70 \mathrm{kbar}$ in a pressure cell [42].

Below about $10 \mathrm{~K}, \mathrm{CePd}_{2} \mathrm{Si}_{2}$ orders antiferromagnetically as shown in Fig. 11(a), with wave vector $\mathbf{Q}=$ $\left[\frac{1}{2} \frac{1}{2} 0\right]$, and a low-temperature ordered moment of about
$0.7 \mu_{B}$ per Ce atom. Its Sommerfeld coefficient is enhanced to about $100 \mathrm{~mJ} / \mathrm{K}^{2} \cdot \mathrm{mol}$. Samples of the present generation show residual resistivity in the $\mu \Omega \cdot \mathrm{cm}$ range 22]. Under hydrostatic pressure of $26 \mathrm{kbar}$, the Néel temperature drops to under $1 \mathrm{~K}$ and, in a pressure window of $\pm 5 \mathrm{kbar}$ around this value, superconductivity appears, with a maximum transition temperature of about $0.4 \mathrm{~K}$ [43]. Curiously enough, normal state resistivity near this pressure follows a temperature dependence, that does not fit the $\rho(T)=\rho_{0}+A T^{2}$ temperature dependence of the Landau Fermi liquid theory, but instead behaves as $\rho(T) \sim T^{1.2}$ over more than a decade in temperature, between about 1 and $40 \mathrm{~K}$ [33].
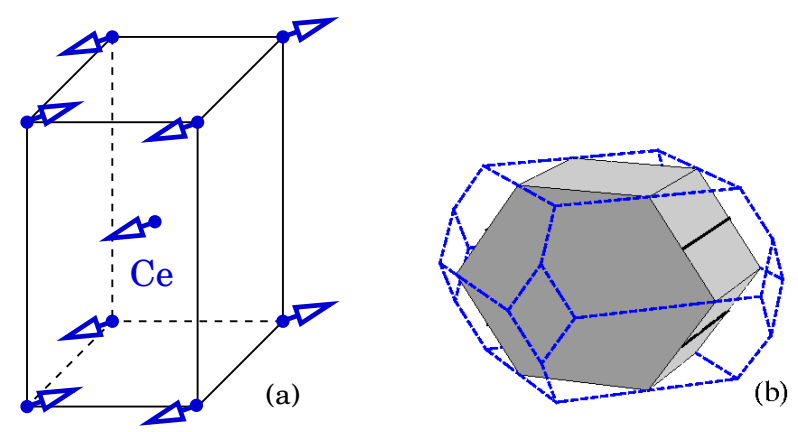

FIG. 11: (color online). Geometry of $\mathrm{CePd}_{2} \mathrm{Si}_{2}$ in real and in reciprocal space. (a) Tetragonal unit cell of $\mathrm{CePd}_{2} \mathrm{Si}_{2}$, showing Ce atoms and the orientation of their magnetic moments. A full sketch, showing $\mathrm{Pd}$ and $\mathrm{Si}$ atoms, is given in [45]. (b) The Brillouin zone boundary of paramagnetic $\mathrm{CePd}_{2} \mathrm{Si}_{2}$ is shown by dashed lines. The shaded hexagonal prism is its antiferromagnetic counterpart.

The unit cell of $\mathrm{CePd}_{2} \mathrm{Si}_{2}$, and its paramagnetic and antiferromagnetic Brillouin zone boundaries are shown in Fig. 11. By symmetry, the degeneracy manifold in a transverse field includes the two hexagonal faces of the MBZ boundary, one of which is shown by darker shading in Fig. 11(b), and the four segments, two of which are shown in black. Along these segments, which are a threedimensional analogue of point $\Sigma$ in Fig. [6(a), another sheet of the degeneracy surface crosses the side faces of the MBZ. According to de Haas-van Alphen (dHvA) experiments [4]], several Fermi surface sheets cross the degeneracy surface. The leading term in the expansion of $g_{\perp}(\mathbf{p})$ around the hexagonal MBZ faces is linear.

$\mathrm{CeRh}_{2} \mathrm{Si}_{2}$ is an isostructural relative of $\mathrm{CePd}_{2} \mathrm{Si}_{2}$, with a modestly enhanced Sommerfeld coefficient of about 23 $\mathrm{mJ} / \mathrm{K}^{2} \cdot \mathrm{mol}$. Between $T_{N 1} \approx 36 \mathrm{~K}$ and $T_{N 2} \approx 25 \mathrm{~K}$, it develops Néel order with $\mathbf{Q}=\left[\frac{1}{2} \frac{1}{2} 0\right]$ [45, 46]. Magnetic structure below $T_{N 2}$ has not yet been established unambiguously [45, 46]. Both $T_{N 1}$ and $T_{N 2}$ drop under pressure [47] and, in an extended pressure window above 5 kbar, $\mathrm{CeRh}_{2} \mathrm{Si}_{2}$ becomes superconducting at a $T_{c}$ with a maximum of about $0.5 \mathrm{~K}$ [8]. Antiferromagnetic structure of $\mathrm{CeRh}_{2} \mathrm{Si}_{2}$ between $T_{N 1}$ and $T_{N 2}$ coincides with that of $\mathrm{CePd}_{2} \mathrm{Si}_{2}$, as does the degeneracy surface in Fig. 
11(b). According to [49], at least one sheet of the Fermi surface of $\mathrm{CeRh}_{2} \mathrm{Si}_{2}$ crosses the degeneracy surface or comes close to it.

\section{Neodymium hexaboride}

Rare earth hexaborides $\mathrm{RB}_{6}$ are an interesting family, whose members show diverse electron and magnetic properties. Of the (relatively) simple ones, $\mathrm{LaB}_{6}$ is a diamagnetic metal, and $\mathrm{SmB}_{6}$ is a mixed valence semiconductor. Of the ordered materials, $\mathrm{EuB}_{6}$ is a ferromagnetic semi-metal, and $\mathrm{CeB}_{6}$ is a heavy fermion metal with at least two ordered phases, whose nature remains to be elucidated after nearly forty years of research.

Three members of the family: $\mathrm{NdB}_{6}, \mathrm{GdB}_{6}$, and $\mathrm{PrB}_{6}$, are antiferromagnetic at low temperature. In $\mathrm{PrB}_{6} \quad$ [50] and in $\mathrm{GdB}_{6}$ [51, 52] alike, two different low-temperature antiferromagnetic states have been found.
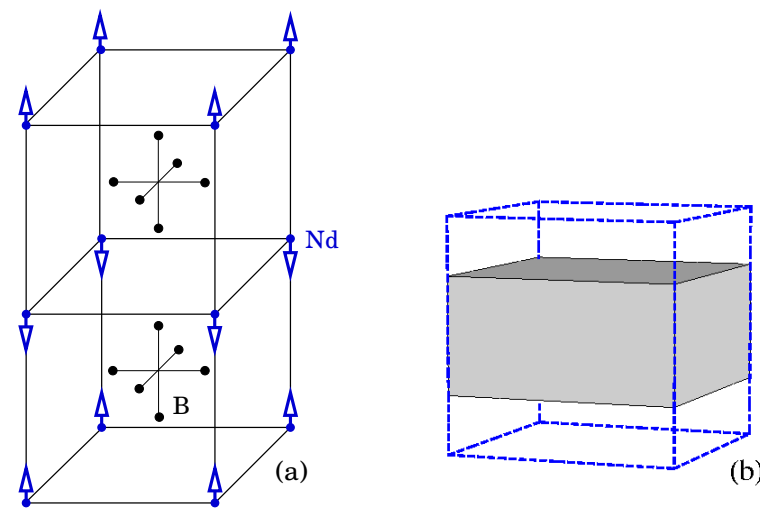

FIG. 12: (color online). Neodymium hexaboride. (a) Crystalline and magnetic structure of $\mathrm{NdB}_{6}$ : a $\mathrm{CsCl}$ structure with $\mathrm{B}$ octahedra replacing $\mathrm{Cl}$ atoms, and $\mathrm{Nd}$ in place of $\mathrm{Cs}$. The arrows show magnetic moments of neodymium atoms. (b) Dashed lines show the cubic Brillouin zone of paramagnetic $\mathrm{NdB}_{6}$. The shaded square prism inside it is the tetragonal Brillouin zone boundary in the antiferromagnetic state. Its darker face denotes the degeneracy plane.

Neodymium hexaboride $\mathrm{NdB}_{6}$ presents a simpler picture: below about $8 \mathrm{~K}$, it is a collinear type-I antiferromagnet with ordering vector $\mathbf{Q}=\left[00 \frac{1}{2}\right]$ and an ordered moment of about $1.74 \mu_{B}$ [53]; antiferromagnetism doubles its cubic unit cell in the $\left[\begin{array}{lll}0 & 0 & 1\end{array}\right]$ direction, as shown in Fig. 12(a). Thus the cubic magnetic Brillouin zone reduces by half in the $\left[\begin{array}{lll}0 & 0 & 1\end{array}\right]$ direction, while keeping its other two dimensions intact, as shown in Fig. 12(b).

In a transverse field, the Kramers degeneracy is protected at the two faces of the MBZ boundary, one of which is shown by darker shading in Fig. 12(b). According to de Haas-van Alphen measurements [54, 55] and to calculations [56], at least one sheet of the Fermi surface crosses the degeneracy surface. Recently studied samples had residual resistivities well below $\mu \Omega \cdot \mathrm{cm}$, and residual resistivity ratios of over a 100 [54, 57].

\section{Other materials of interest}

This subsection contains a brief discussion of other antiferromagnets, where symmetry may protect the degeneracy of special electron states against transverse magnetic field, giving rise to Zeeman spin-orbit coupling (1).

Cuprate superconductors: Electron-doped cuprates such as $\mathrm{Nd}_{2-x} \mathrm{Ce}_{x} \mathrm{CuO}_{4 \pm \delta}$ develop commensurate antiferromagnetic order in a wide range of doping [58], albeit with a modest staggered moment [59]. For such materials, Fig. 6(b) describes the paramagnetic and antiferromagnetic Brillouin zone boundaries. Angle-resolved photoemission experiments [60] on $\mathrm{Nd}_{2-x} \mathrm{Ce}_{x} \mathrm{CuO}_{4 \pm \delta}$ have found carriers in a vicinity of the MBZ boundary. In a transverse magnetic field, these carriers are subject to Zeeman spin-orbit coupling (1), provided antiferromagnetism in the sample is developed well enough.

Recent observation [61] of magnetic oscillations in $\mathrm{YBa}_{2} \mathrm{Cu}_{3} \mathrm{O}_{6.5}$ testifies to great progress in sample quality of cuprates. And the fact that this and other underdoped cuprates are, at the very least, close to commensurate antiferromagnetism, makes them an interesting opportunity to examine the effects of Zeeman spin-orbit coupling.

Borocarbides $\mathrm{RT}_{2} \mathrm{~B}_{2} \mathrm{C}$ with $\mathrm{R}=\mathrm{Sc}, \mathrm{Y}, \mathrm{La}, \mathrm{Th}, \mathrm{Dy}$, Ho, Er, Tm or Lu and $\mathrm{T}=\mathrm{Ni}, \mathrm{Ru}, \mathrm{Pd}$ or Pt have been a subject of active research, driven by interest in interplay between antiferromagnetism and superconductivity [4]. At low temperatures, commensurate antiferromagnetism develops in a number of borocarbides (for instance, in $R \mathrm{Ni}_{2} \mathrm{~B}_{2} \mathrm{C}$ with $R=\mathrm{Pr}$, Dy or Ho), often with a large staggered moment $\left(\approx 8.5 \mu_{B}\right.$ for Dy and Ho) [4]. Zeeman spin-orbit coupling (11) is present whenever a sheet of the Fermi surface crosses the degeneracy manifold, and successful growth of high-quality single crystals [62] makes these materials an interesting case to study.

Organic conductors are an immense and ever growing class of quasi-low-dimensional materials, that show virtually all known types of electron states, found in condensed matter physics [8]. Antiferromagnetism appears in several families of organic conductors, and manifestations of Zeeman spin-orbit coupling (1) are likely to be found in some of them.

Unfortunately, so far nearly all of the information on magnetic structure of organic antiferromagnets has been coming from indirect probes such as magnetic susceptibility measurements [63, 64] and resonance spectroscopies 65, 66, 67]. Neutron diffraction studies are hampered by a typically small ordered moment, and by the difficulties of growing large enough single-crystalline samples. At the moment of writing, I am aware of only a single cycle of neutron scattering experiments [68, 69, 70] 
on an organic conductor. Moreover, in families such as $(\mathrm{TMTSF})_{2}$ Bechgaard salts [8] and $\kappa$-(BEDT-TTF $)_{2} \mathrm{X}$ salts [71, 72], antiferromagnetic states are insulating, and their controlled doping remains a challenge 73 .

With this word of caution, a number of organic conductors may deserve attention. Semi-metallic Bechgaard salt (TMTSF $)_{2} \mathrm{NO}_{3}$ [74], developing a spin density wave state below about $9 \mathrm{~K}$, may be one interesting case. Recently synthesized ethylenedioxytetrathiafulvalenoquinone-1,3diselenolemethide (EDO-TTFVODS), that appears to turn antiferromagnetic below about $4.5 \mathrm{~K}$, and remains normal down to the lowest studied temperature of 0.45 $\mathrm{K}$ [75], may be another. Finally, recent studies [76, 77, 78] of $\left[\mathrm{Au}(\mathrm{tmdt})_{2}\right]$, where tmdt denotes trimethylenetetrathiafulvalenedithiolate, draw attention to this organic conductor. Albeit the material is not yet fully characterized, and its large single crystals remain difficult to grow, it appears to have a Néel temperature of about $110 \mathrm{~K}$ [77, 78], which is anomalously high for an organic material - and shows normal conduction down to at least $10 \mathrm{~K}$.

Heavy fermion materials: Several heavy fermion antiferromagnets were reviewed in detail above. A number of other interesting examples may be found in [10].

Gadolinium antiferromagnets (see [79, 80], and Table I in [81]) offer two important advantages for an experimental study of the Zeeman spin-orbit coupling. Firstly, their often elevated Néel temperature $T_{N}$ (such as $134 \mathrm{~K}$ for $\mathrm{GdAg}$, or $150 \mathrm{~K}$ for $\mathrm{GdCu}$ ) facilitates experimental access to temperatures well below $T_{N}$, where thermal fluctuations of antiferromagnetic order are frozen out. Secondly, large ordered moment of these materials (about $7.5 \mu_{B}$ for $\mathrm{GdAg}$, and about $7.2 \mu_{B}$ for $\mathrm{GdCu}_{2} \mathrm{Si}_{2}$ ) quenches quantum fluctuations. Therefore, gadolinium antiferromagnets fit well into the present framework with its neglect of both quantum and classical fluctuations and shall be convenient for a study of various effects of the Zeeman spin-orbit coupling.

Iron pnictides have been attracting immense attention 82] due to appearance of commensurate antiferromagnetism [7] and high-temperature superconductivity [83] in this copper-free family of materials. Combination of commensurate antiferromagnetism [7] with essentially metallic normal state conduction [83, 84] not only contrasts iron pnictides with the cuprates (that are believed to be Mott insulators), but also makes the former materials likely to manifest a substantial momentum dependence of the $g$-tensor.

\section{DISCUSSION}

\section{Effects of relativistic spin-orbit coupling}

The arguments above appealed to the exchange symmetry approximation [17]: the point symmetry opera- tions of the electron Hamiltonian in an antiferromagnet were considered inert with respect to spin, and the common relativistic spin-orbit coupling, that appears in the absence of an external magnetic field, was thus neglected. I will now examine the effects it may have.

Firstly, this spin-orbit interaction generates magnetic anisotropy, that creates a preferential orientation of the staggered magnetization $\mathbf{n}$ with respect to the crystal axes. In an experiment, this allows one to vary the magnetic field orientation with respect to $\mathbf{n}$ as long as the field remains below the reorientation threshold.

At the same time, the spin-orbit coupling may eliminate those spatial symmetries, that rotate the magnetization density with respect to the lattice. For instance, certain spin rotations and spatial transformations, that were independent symmetries within the exchange symmetry approximation, may survive only when combined. I will now illustrate this by two examples of Section IV.

A simple case of the spin-orbit coupling affecting the Kramers degeneracy manifold in a transverse field is given by a two-dimensional antiferromagnet on a squaresymmetry lattice as in Fig. 6(b). Here, the Kramers degeneracy at the antiferromagnetic Brillouin zone boundary relies, everywhere except for points $\Sigma$, on the symmetry with respect to reflections in diagonal planes 1 and 2. If either of these reflections changes the orientation of $\boldsymbol{\Delta}_{\mathbf{r}}$ with respect to the crystal axes, a spin-orbit coupling may lift the degeneracy at a relevant part of the magnetic Brillouin zone boundary, except for points $\Sigma$. However, if the magnetization density points along one of these diagonal axes, the degeneracy survives at the two faces of the MBZ boundary, that are normal to this axis.

In the case of commensurate order in chromium, consider a single-domain sample with magnetic structure shown in Fig. 7. For a Bloch state $|\mathbf{p}\rangle$ with a momentum $\mathbf{p}$ at one of the two horizontal faces of the magnetic Brillouin zone in Fig 8(a), the degenerate partner state $\theta T_{a} U_{n}(\pi)|\mathbf{p}\rangle$ has momentum $-\mathbf{p}$ at the other horizontal face of the MBZ. Coordinate rotation by $\pi$ around the vertical symmetry axis, passing through the center $\Gamma$ of the Brillouin zone, transforms the momentum $\mathbf{p}$ into $\mathbf{p}+\mathbf{Q}$, equivalent to $\mathbf{p}$ up to reciprocal lattice vector $\mathbf{Q}$ of the antiferromagnetic state.

By contrast, for a momentum $\mathbf{p}$ at one of the vertical faces of the MBZ, coordinate rotation by $\pi$ around a horizontal axis is required, and such a rotation inverts $\boldsymbol{\Delta}_{\mathbf{r}}$ once the latter is attached to the crystal axes. Thus spin-orbit coupling tends to lift the degeneracy at the vertical faces of the MBZ, leaving it intact at the two horizontal faces. The other examples of Section III can be analyzed similarly.

Finally, those spin-orbit coupling terms, that act directly on the electron spin and tend to lift the double degeneracy of Bloch eigenstates even in the absence of magnetic field, were neglected here altogether. 


\section{Relation to earlier work}

When symmetries of a system involve time reversal alone or in combination with other operations - a proper treatment must involve non-unitary symmetry groups: those containing unitary as well as anti-unitary elements. In this case, construction of irreducible representations is complicated by the fact that anti-unitary elements involve complex conjugation. In a group representation, combination of two unitary elements $u_{1}$ and $u_{2}$ is represented by the product of the corresponding matrices $\mathbf{D}\left(u_{1}\right)$ and $\mathbf{D}\left(u_{2}\right): \mathbf{D}\left(u_{1} u_{2}\right)=\mathbf{D}\left(u_{1}\right) \mathbf{D}\left(u_{2}\right)$. By contrast, combination of an anti-unitary element $a$ with a unitary element $u$ involves complex conjugation: $\mathbf{D}(a u)=$ $\mathbf{D}(a) \mathbf{D}^{*}(u)$. As a result, irreducible representations of a non-unitary group must include a unitary representation and its complex conjugate on an equal footing. Discussion of such representations (called co-representations) was given by Wigner, along with the analysis of arising possibilities with the help of the Frobenius-Schur criterion [85]. Later, Herring studied spectral degeneracies, emerging in crystals due to time reversal symmetry and, among other things, extended this criterion to space groups [86]. In a subsequent work, Dimmock and Wheeler generalized the criterion further, to magnetic crystals, and pointed out the sufficient condition (6) for the appearance of extra degeneracies [13].

The present work identifies the symmetry, that protects the Kramers degeneracy in a Néel antiferromagnet against transverse magnetic field, as a conspiracy between the anti-unitary symmetry $\mathbf{U}_{\mathbf{n}}(\pi) \mathbf{T}_{\mathbf{a}} \theta$, inherent to any collinear commensurate antiferromagnet in a transverse field, and the crystal symmetry of those special momenta at the MBZ boundary, that are defined by Eqn. (6). Formally, the present work is an extension of [13], since one may think of the last two terms in (4) as of the exchange field of a fictitious magnetic crystal in zero field. However, Kramers degeneracy in a magnetic field has rather special and remarkable experimental signatures, some of which are outlined at the end of this section.

Last but not the least, Ref. 14] was an important source of inspiration for the present work. Its authors studied the electron eigenstates in a Néel antiferromagnet on a lattice of square symmetry and, for this particular case, pointed out the disappearance of $g_{\perp}(\mathbf{p})$ at the MBZ boundary, as well as the ensuing substantial momentum dependence of $g_{\perp}$ in the Zeeman coupling (1). The present article builds on Ref. [14] by elucidating the structure of the manifold of degenerate states for an arbitrary crystal symmetry, and for an arbitrary transverse field that can be sustained by the antiferromagnet before its sublattices collapse. This is to be contrasted with the analysis of Ref. 14], performed to the linear order in the field. Several other aspects of Ref. [14] are discussed in Appendix D.

\section{Experimental signatures}

The Kramers degeneracy at special momenta on the MBZ boundary and the resultant Zeeman spin-orbit coupling have a number of interesting consequences. For instance, a substantial momentum dependence of $g_{\perp}(\mathbf{p})$ in Eqn. (1) means that, generally, the Electron Spin Resonance (ESR) frequency of a carrier in the vicinity of the degeneracy manifold varies along the quasiclassical trajectory in momentum space.

For a weakly-doped antiferromagnetic insulator with a conduction band minimum on the degeneracy manifold, this leads to an inherent broadening of the ESR line with doping and, eventually, complete loss of the ESR signal. In fact, this may well be the reason behind the long-known 'ESR silence' [87] of the cuprates. Suppression of Pauli paramagnetism in the transverse direction with respect to staggered magnetization is another simple consequence of vanishing $g_{\perp}(\mathbf{p})$.

At the same time, a momentum dependence of $g_{\perp}(\mathbf{p})$ allows excitation of spin resonance transitions by AC electric rather than magnetic field [88, 89] - a vivid effect of great promise for controlled spin manipulation, currently much sought after in spin electronics. Its absorption matrix elements are defined by $\boldsymbol{\Xi}_{\mathrm{p}}$ of Eqn. (19). Comparison with Eqn. (11) shows that, within the weakcoupling model (8), $\Xi_{\mathbf{p}} / \hbar$ is of the order of the antiferromagnetic coherence length $\xi \sim \frac{\hbar v_{F}}{\Delta}$, and may be of the order of the lattice period or much greater. By contrast, the ESR matrix elements are defined by the Compton length $\lambda_{C}=\frac{\hbar}{m c} \approx 0.4 \mathrm{pm}$. Thus, matrix elements of electrically excited spin transitions exceed those of ESR by about $\frac{\hbar c}{e^{2}} \cdot \frac{\epsilon_{F}}{\Delta} \approx 137 \cdot \frac{\epsilon_{F}}{\Delta}$, or at least by two orders of magnitude. Being proportional to the square of the appropriate transition matrix element, resonance absorption due to electric excitation of spin transitions exceeds that of ESR at least by four orders of magnitude.

Last but not the least - according to Eqn. (19), resonance absorption in this phenomenon shows a non-trivial dependence on the orientation of the $\mathrm{AC}$ electric field with respect to the crystal axes, and on the orientation of the DC magnetic field with respect to the staggered magnetization.

The Zeeman spin-orbit coupling may also manifest itself in other experiments on antiferromagnetic conductors. In particular, de Haas-van Alphen oscillations 90] and magneto-optical response may be modified. In various types of electron response, interesting effects may arise due to an extra term $\mathbf{v}_{Z S O}$ in the electron velocity operator, emerging due to a substantial momentum dependence of $g_{\perp}(\mathbf{p})$ in Eqn. (1):

$$
\mathbf{v}_{Z S O}=\nabla_{\mathbf{p}} \mathcal{H}_{Z S O}=-\mu_{B} \nabla_{\mathbf{p}} g_{\perp}(\mathbf{p})\left(\mathbf{H}_{\perp} \cdot \boldsymbol{\sigma}\right) .
$$

This term describes spin current. However, $g_{\perp}(\mathbf{p})$ is even in $\mathbf{p}$ due to inversion symmetry and thus, in equilibrium, 
the net spin current must vanish. This may change, if the system were tilted, say, by electric current or otherwise - however, the resulting effect would be proportional to the 'tilt' and, in addition to this, would be small in the measure of $H_{\perp} / \Delta$.

\section{Conclusions}

In this work, I studied the degeneracy of electron Bloch states in a Néel antiferromagnet, subject to a transverse magnetic field - and described the special points in momentum space, where the degeneracy is protected by a hidden anti-unitary symmetry.

I discussed the simplest properties and some of the manifestations of the Zeeman spin-orbit coupling, arising in a magnetic field due to this degeneracy, and outlined several examples of interesting materials, where such a coupling may be present. Finally, I reviewed the results and their relation to earlier work.

The degeneracy of special Bloch states in a transverse field hinges only on the symmetry of the antiferromagnetic state, and thus holds in weakly coupled and strongly correlated materials alike - provided long-range antiferromagnetic order and well-defined electron quasiparticles are present. Under these conditions, thermal and quantum fluctuations of the antiferromagnetic order primarily renormalize the sublattice magnetization, leaving intact the degeneracy of special electron states in a transverse field - certainly in the leading order in fluctuations. Detailed account of fluctuations is outside the scope of this article.

\section{ACKNOWLEDGMENTS}

I am indebted to S. Brazovskii and G. Shlyapnikov for inviting me to Orsay, to LPTMS for the kind hospitality, and to IFRAF for generous support. I am grateful to S. Brazovskii, M. Kartsovnik and K. Kanoda for references on organic antiferromagnets, and to C. Capan for drawing my attention to gadolinium compounds. It is my pleasure to thank S. Carr, N. Cooper, N. Shannon, and M. Zhitomirsky for their helpful comments on the manuscript, and A. Chubukov and G. Volovik for enlightening discussions.

\section{APPENDIX A: ORTHOGONALITY RELATION}

This Appendix proves the relation

$$
\left\langle\phi\left|[\mathcal{O} \theta]^{+}\right|[\mathcal{O} \theta] \mid \psi\right\rangle=\langle\psi \mid \phi\rangle,
$$

where $|\phi\rangle$ and $|\psi\rangle$ are arbitrary states, $\mathcal{O}$ is an arbitrary unitary operator, and $\theta$ is time reversal. In the main text, this relation is used for $|\phi\rangle=\mathcal{O} \theta|\psi\rangle$; in this case, when read right to left, Eqn. (21) yields

$$
\langle\psi|\mathcal{O} \theta| \psi\rangle=\left\langle\psi\left|\left[(\mathcal{O} \theta)^{+}\right]^{2}\right|(\mathcal{O} \theta) \mid \psi\right\rangle .
$$

Whenever $|\psi\rangle$ is an eigenvector of the linear operator $[\mathcal{O} \theta]^{2}$ with an eigenvalue different from unity, Eqn. (22) proves orthogonality of $|\psi\rangle$ and $\mathcal{O} \theta|\psi\rangle$.

The proof of Eqn. (21) is based on the obvious relation $(\mathcal{C} \phi, \mathcal{C} \psi)=(\psi, \phi)$ for arbitrary complex vectors $\phi$ and $\psi$, where $(\psi, \phi) \equiv \sum_{i} \psi_{i}^{*} \phi_{i}$ denotes scalar product, and $\mathcal{C}$ is complex conjugation. Hence, for an arbitrary unitary operator $\mathcal{O}$, one finds $(\mathcal{O C} \phi, \mathcal{O C} \psi)=(\psi, \phi)$, due to invariance of scalar product under unitary transformation. Time reversal $\theta$ can be presented as a product of $\mathcal{C}$ and a unitary operator 85]: $\theta=\mathcal{V C}$, thus $\mathcal{C}=\mathcal{V}^{-1} \theta$ and, therefore, $(\mathcal{O} \theta \phi, \mathcal{O} \theta \psi)=(\psi, \phi)$. As a result, for arbitrary states $|\psi\rangle$ and $|\phi\rangle$, one finds $\left\langle\phi\left|[\mathcal{O} \theta]^{+}\right|[\mathcal{O} \theta] \mid \psi\right\rangle=\langle\psi \mid \phi\rangle$, which indeed amounts to (21).

\section{APPENDIX B: CANTING OF THE SUBLATTICES}

Canting of the two sublattices by transverse field $\mathbf{H}_{\perp}$ induces a component $\boldsymbol{\Delta}_{\mathbf{r}}^{\perp}$ of the magnetization density along the field, with the periodicity of the underlying lattice: $\boldsymbol{\Delta}_{\mathbf{r}+\mathbf{a}}^{\perp}\left(H_{\perp}\right)=\boldsymbol{\Delta}_{\mathbf{r}}^{\perp}\left(H_{\perp}\right)$, as shown in Fig. 3. As a result, the diagonal part of Hamiltonian (8) acquires an additional term $\left(\boldsymbol{\Delta}_{\mathbf{p}}^{\perp} \cdot \boldsymbol{\sigma}\right)$, and Hamiltonian (8) thus takes the form

$$
\mathcal{H}=\left[\begin{array}{cc}
\epsilon_{\mathbf{p}}-\left(\tilde{\Delta}_{\mathbf{p}}^{\perp} \cdot \boldsymbol{\sigma}\right) & \left(\boldsymbol{\Delta}_{\|} \cdot \boldsymbol{\sigma}\right) \\
\left(\boldsymbol{\Delta}_{\|} \cdot \boldsymbol{\sigma}\right) & \epsilon_{\mathbf{p}+\mathbf{Q}}-\left(\tilde{\boldsymbol{\Delta}}_{\mathbf{p}+\mathbf{Q}}^{\perp} \cdot \boldsymbol{\sigma}\right)
\end{array}\right],
$$

where $\tilde{\boldsymbol{\Delta}}_{\mathbf{p}}^{\perp} \equiv \mathbf{H}_{\perp}+\boldsymbol{\Delta}_{\mathbf{p}}^{\perp}$.

The same choice of spin axes as in Section III splits Hamiltonian (23) into two independent pieces

$$
\mathcal{H}_{1(2)}=\left[\begin{array}{cc}
\epsilon_{\mathbf{p}} \mp \tilde{\Delta}_{\mathbf{p}}^{\perp} & \Delta_{\|} \\
\Delta_{\|} & \epsilon_{\mathbf{p}+\mathbf{Q}} \pm \tilde{\Delta}_{\mathbf{p}+\mathbf{Q}}^{\perp}
\end{array}\right] .
$$

As in Section III, momentum shift by $\mathbf{Q}$ maps $\mathcal{H}_{1}$ and $\mathcal{H}_{2}$ onto each other, and spectral symmetries of Hamiltonian (23) coincide with those discussed in the second subsection of Section III. Thus all of the conclusions of Section III remain valid after sublattice canting is accounted for.

However, while degeneracy at special points is protected by symmetry, the shape of the manifold of degenerate states may change under various perturbations. For instance, sublattice canting in a transverse field modifies the equation, describing this manifold and, for the conduction band, turns it into

$$
\psi_{\mathbf{p}}+\frac{\phi_{\mathbf{p}} \zeta_{\mathbf{p}}}{\sqrt{\Delta_{\|}^{2}+\phi_{\mathbf{p}}^{2}+\zeta_{\mathbf{p}}^{2}}},
$$


where $\phi_{\mathbf{p}} \equiv \frac{1}{2}\left[\tilde{\Delta}_{\mathbf{p}}^{\perp}+\tilde{\Delta}_{\mathbf{p}+\mathbf{Q}}^{\perp}\right]$ and $\psi_{\mathbf{p}} \equiv \frac{1}{2}\left[\tilde{\Delta}_{\mathbf{p}}^{\perp}-\tilde{\Delta}_{\mathbf{p}+\mathbf{Q}}^{\perp}\right]$. Since $\boldsymbol{\Delta}_{\mathbf{r}}^{\perp}$ has the real-space periodicity of the paramagnetic state, $\tilde{\Delta}_{\mathbf{p}}^{\perp}$ enjoys the same reciprocal space symmetry as $\epsilon_{\mathbf{p}}$. In particular, $\tilde{\Delta}_{\mathbf{p}+2 \mathbf{Q}}^{\perp}=\tilde{\Delta}_{\mathbf{p}}^{\perp}$, and $\tilde{\Delta}_{\mathbf{p}}^{\perp}=\tilde{\Delta}_{-\mathbf{p}}^{\perp}$ (the latter property is also protected by the $\mathbf{U}_{\mathbf{l}} \theta$ symmetry). At the same time, $\psi_{\mathbf{p}+\mathbf{Q}}=-\psi_{\mathbf{p}}$, and $\phi_{\mathbf{p}+\mathbf{Q}}=\phi_{\mathbf{p}}$; thus the symmetry-dictated degeneracy points such as $\mathbf{p}=\frac{\mathbf{Q}}{2}$ explicitly belong to the manifold of Eqn. (25), as they should.

In the limit of vanishing $H_{\perp}, \tilde{\Delta}_{\mathbf{p}}^{\perp}$ is linear in the field: $\tilde{\Delta}_{\mathbf{p}}^{\perp}=H_{\perp}\left[1+\chi_{\mathbf{p}}^{\perp}\right]$, where $\chi_{\mathbf{p}}^{\perp}$ describes microscopic transverse susceptibility of the antiferromagnet. Now one may expand Eqn. (25) to linear order in the field to obtain the following equation for the degeneracy manifold:

$$
\chi_{\mathbf{p}}^{-}+\frac{\chi_{\mathbf{p}}^{+} \zeta_{\mathbf{p}}}{\sqrt{\Delta_{\|}^{2}+\zeta_{\mathbf{p}}^{2}}}=0,
$$

where $\chi_{\mathbf{p}}^{ \pm} \equiv \chi_{\mathbf{p}} \pm \chi_{\mathbf{p}+\mathbf{Q}}$. Compared with equation $\zeta_{\mathbf{p}}=0$ of Section III, the sublattice canting affects the degeneracy manifold already in zeroeth order in $H_{\perp}$.

\section{APPENDIX C: DIMENSIONALITY OF THE DEGENERACY MANIFOLD}

The dimensionality of the degeneracy manifold in a transverse field is one less than that of the momentum space for simple reasons, that rely only on the symmetry of the antiferromagnetic state. According to Eqn. (3), zero-field Bloch eigenstates $|1\rangle \equiv|\mathbf{p}\rangle$ and $|2\rangle \equiv \mathcal{I} \mathbf{T}_{\mathbf{a}} \theta|\mathbf{p}\rangle$ form a Kramers doublet at momentum p. Its splitting $\delta \mathcal{E}(\mathbf{p})$ in a transverse field $\mathbf{H}_{\perp}$ is given by

$$
\delta \mathcal{E}(\mathbf{p})=2 \sqrt{\left|V_{12}(\mathbf{p})\right|^{2}+\frac{1}{4}\left[V_{11}(\mathbf{p})-V_{22}(\mathbf{p})\right]^{2}},
$$

where $V_{i j}(\mathbf{p}) \equiv\left\langle i\left|\left(\mathbf{H}_{\perp} \cdot \boldsymbol{\sigma}\right)\right| j\right\rangle$. Magnetic field being uniform, $\left(\mathbf{H}_{\perp} \cdot \boldsymbol{\sigma}\right)$ commutes with $\mathcal{I} \mathbf{T}_{\mathbf{a}}$; it also changes sign under time reversal. Thus, $V_{22}(\mathbf{p})=-V_{11}(\mathbf{p})$. At the same time, the off-diagonal matrix element $V_{12}(\mathbf{p})$ vanishes identically:

$$
\begin{aligned}
& \left\langle\mathbf{p}\left|\left(\mathbf{H}_{\perp} \cdot \boldsymbol{\sigma}\right) \mathcal{I} \mathbf{T}_{\mathbf{a}} \theta\right| \mathbf{p}\right\rangle=\sum_{\mathbf{q}}\left\langle\mathbf{p}\left|\left(\mathbf{H}_{\perp} \cdot \boldsymbol{\sigma}\right)\right| \mathbf{q}\right\rangle\left\langle\mathbf{q}\left|\mathcal{I} \mathbf{T}_{\mathbf{a}} \theta\right| \mathbf{p}\right\rangle= \\
= & \sum_{\mathbf{q}} V_{11}(\mathbf{p}) \delta_{\mathbf{p q}}\left\langle\mathbf{q}\left|\mathcal{I} \mathbf{T}_{\mathbf{a}} \theta\right| \mathbf{p}\right\rangle=V_{11}(\mathbf{p})\left\langle\mathbf{p}\left|\mathcal{I} \mathbf{T}_{\mathbf{a}} \theta\right| \mathbf{p}\right\rangle \equiv 0,(28
\end{aligned}
$$

where insertion of unity $\mathbf{1}=\sum_{\mathbf{q}}|\mathbf{q}\rangle\langle\mathbf{q}|$ was used in the first line, uniformity of $\mathbf{H}_{\perp}$ in the second, and the final equality followed from Eqn. (3). Therefore,

$$
\delta \mathcal{E}(\mathbf{p})=2\left|V_{11}(\mathbf{p})\right|
$$

and, barring a special case, equation $\delta \mathcal{E}(\mathbf{p})=0$ defines a $(d-1)$-dimensional surface of zero $g_{\perp}(\mathbf{p})$ in $d$ dimensional momentum space. The Kramers degeneracy subset contains, at the very least, the star of the momentum $\mathbf{p}=\mathbf{Q} / 2$ [see Eqn.(6) and the subsequent discussion], and the $(\mathbf{k} \cdot \mathbf{p})$ expansion 91] around these points shows, that they are not isolated, but rather belong to a $(d-1)$-dimensional manifold. The latter is continuous, with the obvious exception of $d=1$.

Finally, notice that, according to (29),$\delta \mathcal{E}(\mathbf{p})$ is periodic with the antiferromagnetic ordering wave vector $\mathbf{Q}$ :

$$
\delta \mathcal{E}(\mathbf{p}+\mathbf{Q})=\delta \mathcal{E}(\mathbf{p}),
$$

thanks to $\mathbf{Q}$ being a reciprocal lattice vector in the antiferromagnetic state. Therefore, properties (16) and (17) are indeed model-independent, as opposed to hinging on an approximation of the weak-coupling model (8).

\section{APPENDIX D: REVISITING [14]}

In Ref. [14], Brazovskii and Lukyanchuk stated that operator $\boldsymbol{\Lambda}=(\mathbf{n} \cdot \boldsymbol{\sigma})$ exchanges the momenta $\mathbf{p}$ and $\mathbf{p}+\mathbf{Q}$ in (8), and thus represents the momentum boost by the ordering wave vector $\mathbf{Q}$ in reciprocal space. In a commensurate antiferromagnetic state, $\mathbf{Q}$ becomes a reciprocal lattice vector, and thus $\boldsymbol{\Lambda}$ must be a symmetry of the Hamiltonian. With the assumption of the effective Zeeman coupling (1), this lead the authors of Ref. 14] to the relation $g_{\perp}(\mathbf{p}+\mathbf{Q})=-g_{\perp}(\mathbf{p})($ Eqn. (16) of the present work), and to the conclusion that $g_{\perp}(\mathbf{p})=0$ at the MBZ boundary. Unfortunately, while this beautiful result is indeed correct for a lattice of square symmetry, several circumstances prevent one from embracing these arguments.

Most importantly, they hinge solely on the symmetry under translation by $\mathbf{Q}$, put otherwise - on commensurability of magnetic order with the crystal lattice. If correct, this would imply that, in an arbitrary commensurate Néel antiferromagnet, Kramers degeneracy takes place at the entire MBZ boundary regardless of the underlying crystal symmetry. The toy example (18) with $\eta \neq 1$ shows, that this is not at all necessarily the case.

Indeed, for a generic crystal symmetry, the condition $g_{\perp}(\mathbf{p}+\mathbf{Q})=-g_{\perp}(\mathbf{p})$ (see [14] and Eqn. (16) of the present work) does not, by itself, restrict the manifold $g_{\perp}(\mathbf{p})=0$ to the MBZ boundary. However, the 'Kramers' subset of the manifold of degenerate states can be obtained by combining Eqn. (16) with the crystal symmetries (see the subsection on spectral symmetries in momentum space in Section III). For instance, combined with the inversion symmetry $g_{\perp}(-\mathbf{p})=g_{\perp}(\mathbf{p})$, Eqn. (16) stipulates that $g_{\perp}(\mathbf{Q} / 2)=0$. Similarly, disappearance of $g_{\perp}(\mathbf{p})$ at the entire MBZ boundary for the square symmetry case can be obtained by using Eqn. (16) and the point symmetries of the square lattice. For a finite as opposed to infinitesimal field, these results were established in Section II and in the first two examples in Section IV. 
On a more technical level, the operator $\boldsymbol{\Lambda}$ is equivalent to $\mathbf{U}_{\mathbf{n}}(\pi)$ and thus inverts the sign of the transverse component of the field. Hence, in a field with non-zero transverse component $\mathbf{H}_{\perp}, \boldsymbol{\Lambda}$ ceases to be a symmetry of the Hamiltonian, in agreement with the first line of Table I- and thus can no longer represent the momentum boost by $\mathbf{Q}$.

* Present address: Department of Physics and Astronomy, University of South Carolina, Columbia, SC 29208, USA.

[1] E. Fawcett, Rev. Mod. Phys. 60, 209 (1988).

[2] E. Fawcett, H. L. Alberts, V. Yu. Galkin, D. R. Noakes, J. V. Yakhmi, Rev. Mod. Phys. 66, 25 (1994).

[3] N. I. Kulikov, V. V. Tugushev, Sov. Phys. Uspekhi 27, 954 (1984).

[4] K-H. Müller and V. N. Narozhnyi, Rep. Prog. Phys. 64, 943 (2001).

[5] Robert J. Birgeneau, Chris Stock, John M. Tranquada and Kazuyoshi Yamada, Journ. Phys. Soc. Jpn. 75, 111003 (2006).

[6] John M. Tranquada, in Handbook of High-Temperature Superconductivity, edited by J. Robert Schrieffer and James S. Brooks (Springer, New York 2007).

[7] Clarina de la Cruz et al., Nature 453, 899 (2008).

[8] P. M. Chaikin, J. Phys. I France 6, 1875 (1996).

[9] Y. Kuramoto and Y. Kitaoka, Dynamics of Heavy Electrons (Clarendon Press, Oxford, 2000).

[10] R. A. Robinson, in Magnetism in Heavy Fermion Systems, edited by Harry B. Radousky (World Scientific 2000).

[11] J. Flouquet, arXiv:cond-mat/0501602 (2005).

[12] C. Herring, in Magnetism, edited by George T. Rado and Harry Suhl (Academic Press, New York and London, 1966), Vol. IV, Chap. XIII.

[13] J. O. Dimmock and R. G. Wheeler, Phys. Rev. 127, 391 (1962).

[14] S. A. Brazovskii and I. A. Lukyanchuk, Zh. Eksp. Teor. Fiz. 96, 2088 (1989) [Sov. Phys. JETP 69, 1180 (1989)].

[15] S. A. Brazovskii, I. A. Lukyanchuk, and R. R. Ramazashvili, Jr., Pisma Zh. Eksp. Teor. Fiz. 49, 557 (1989) [JETP Lett. 49, 644 (1989)].

[16] Revaz Ramazashvili, Phys. Rev. Lett. 101, 137202 (2008).

[17] A. F. Andreev, V. I. Marchenko, Usp. Fiz. Nauk 130, 39 (1980) [Sov. Phys. Uspekhi 23, 21 (1980)].

[18] Hartmut Zabel, J. Phys.: Condens. Matter 11, 9303 (1999).

[19] J. M. Lawrence and S. M. Shapiro, Phys. Rev. B 22, 4379 (1980).

[20] G. Knebel, D. Braithwaite, P. C. Canfield, G. Lapertot, and J. Flouquet, Phys. Rev. B 65, 024425 (2001).

[21] Takao Ebihara, N. Harrison, M. Jaime, Shinya Uji, and J. C. Lashley, Phys. Rev. Lett. 93, 246401 (2004).

[22] F. M. Grosche, I. R. Walker, S. R. Julian, N. D. Mathur, D. M. Freye, M. J. Steiner and G. G. Lonzarich, J. Phys.: Condens. Matter 13, 2845 (2001).

[23] P. Dervenagas, D. Kaczorowski, F. Bourdarot, P. Burlet, A. Czopnik, G.H. Lander, Physica B 269, 368 (1999).

[24] M. H. van Maaren, H. J. van Daal, K. H. J. Buschow, and C. J. Schinkel, Sol. St. Comm. 14, 145 (1974).

[25] A. L. Cornelius, A. J. Arko, J. L. Sarrao, J. D. Thompson, M. F. Hundley, C. H. Booth, N. Harrison, P. M. Oppeneer, Phys. Rev. B 59, 14473 (1999).

[26] Dai Aoki, Norihito Suzuki, Kousaku Miyake, Yoshihiko Inada, Rikio Settai, Kiyohiro Sugiyama, Etsuji Yamamoto, Yoshinori Haga, Yoshichika Onuki, Tetsutaro Inoue, Koichi Kindo, Hitoshi Sugawara, Hideyuki Sato and Hiroshi Yamagami, Journ. Phys. Soc. Jpn. 70, 538 (2001).

[27] A. Murasik, J. Leciejewicz, S. Ligenza, and A. Misiuk, Phys. Stat. Sol. A 20, 395 (1973).

[28] Yoshihumi Tokiwa, Dai Aoki, Yoshinori Haga, Etuji Yamamoto, Shugo Ikeda, Rikio Settai, Akio Nakamura and Yoshichika Ōnuki, Journ. Phys. Soc. Jpn. 70, 3326 (2001).

[29] Jon Lawrence, Phys. Rev. B 20, 3770 (1979).

[30] S. Nasu, A. M. van Diepen, H. H. Neumann, and R. S. Craig, J. Phys. Chem. Solids 32, 2773 (1971).

[31] I. R. Walker, F. M. Grosche, D. M. Freye and G. G. Lonzarich, Physica C 282-287, 303 (1997).

[32] N. D. Mathur, F. M. Grosche, S. R. Julian, I. R. Walker, D. M. Freye, R. K. W. Haselwimmer \& G. G. Lonzarich, Nature 394, 39 (1998).

[33] M. Endo, N. Kimura, H. Aoki, T. Terashima, S. Uji, T. Matsumoto, and T. Ebihara, Phys. Rev. Lett. 93, 247003 (2004).

[34] M. Biasini and G. Ferro, A. Czopnik, Phys. Rev. B 68, 094513 (2003).

[35] L. P. Gor'kov and P. D. Grigoriev, Phys. Rev. B 73, 060401(R) (2006).

[36] Takao Ebihara, Izuru Umehara, Ariane Keiko Albessard, Kazuhiko Satoh and Yoshichika Ōnuki, J. Phys. Soc. Jpn. 61, 1473 (1992).

[37] Ján Rusz, Maurizio Biasini, Phys. Rev. B 71, 233103 (2005).

[38] N. A. Curry, Proc. Phys. Soc. 86, 1193 (1965).

[39] M. Nakashima, Y. Haga, E. Yamamoto, Y. Tokiwa, M. Hedo, Y. Uwatoko, R. Settai and Y. Ōnuki, J. Phys.: Condens. Matter 15, S2007 (2003).

[40] F. Steglich, J. Aarts, C. D. Bredl, W. Lieke, D. Meschede, W. Franz, H. Schäfer, Phys. Rev. Lett. 43, 1892 (1979).

[41] G. Knopp, A. Loidl, K. Knorr, L. Pawlak, M. Duczmal, R. Caspary, U. Gottwick, H. Spille, F. Steglich, and A.P. Murani, Z. Phys. B - Condensed Matter 77, 95 (1989).

[42] D. Jaccard, K. Behnia and J. Sierro, Physics Letters A 163 (1992) 475480.

[43] F. M. Grosche, S. R. Julian, N. D. Mathur, G. G. Lonzarich, Physica B 223 \& 224, 50 (1996).

[44] I. Sheikin, A. Gröger, S. Raymond, D. Jaccard, D. Aoki, H. Harima, and J. Flouquet, Phys. Rev. B 67, 094420 (2003).

[45] B. H. Grier, J. M. Lawrence, V. Murgai, and R. D. Parks, Phys. Rev. B 29, 2664 (1984).

[46] Shuzo Kawarazaki, Masugu Sato, Yoshihito Miyako, Nobusato Chigusa, Kenji Watanabe, Naoto Metoki, Yoshihiro Koike, Masakazu Nishi, Phys. Rev. B 61, 4167 (2000).

[47] Masashi Ohashi, Gendo Oomi, Sadayoshi Koiwai, Masato Hedo and Yoshiya Uwatoko, Phys. Rev. B 68, 144428 (2003).

[48] R. Movshovich, T. Graf, D. Mandrus, J. D. Thompson, J. L. Smith, and Z. Fisk, Phys. Rev. B 53, 8241 (1996).

[49] S. Araki, R. Settai, T. C. Kobayashi, H. Harima, and Y. 
Ōnuki, Phys. Rev. B 64, 224417 (2001).

[50] P. Burlet, J. M. Effantin, J. Rossat-Mignod, S. Kunii and T. Kasuya, Journ. de Phys. Colloque C8, 49, 459 (1988).

[51] R. M. Galera, D. P. Osterman, J. D. Axe, S. Kunii, and T. Kasuya, J. Appl. Phys. 63, 3580 (1988).

[52] D.F. McMorrow, K.A. McEwen, J-G. Park, S. Lee, D. Mannix, F. Iga, T. Takabatake, Physica B 345, 66 (2004).

[53] C. M. McCarthy and C. W. Tompson, J. Phys. Chem. Solids 41, 1319 (1980).

[54] Y. Onuki, A. Umezawa, W. K. Kwok, G. W. Crabtree, M. Nishihara, T. Yamazaki, T. Omi, and T. Komatsubara, Phys. Rev. B 40, 11195 (1989).

[55] R. G. Goodrich, N. Harrison, and Z. Fisk, Phys. Rev. Lett. 97, 146404 (2006).

[56] Yasunori Kubo, Setsuro Asano, Hisatomo Harima, and Akira Yanase, Journ. Phys. Soc. Jpn. 62, 205 (1993).

[57] Jolanta Stankiewicz, Satoru Nakatsuji, Zachary Fisk, Phys. Rev. B 71, 134426 (2005).

[58] E. M. Motoyama, G. Yu, I. M. Vishik, O. P. Vajk, P. K. Mang \& M. Greven, Nature 445, 186 (2007).

[59] M. Matsuda, Y. Endoh, K. Yamada, H. Kojima, I. Tanaka, R. J. Birgeneau, M. A. Kastner, G. Shirane, Phys. Rev. B 45, 12548 (1992).

[60] N. P. Armitage et al., Phys. Rev. Lett. 88, 257001 (2002).

[61] Nicolas Doiron-Leyraud, Cyril Proust, David LeBoeuf, Julien Levallois, Jean-Baptiste Bonnemaison, Ruixing Liang, D. A. Bonn, W. N. Hardy \& Louis Taillefer, Nature 447, 565 (2007).

[62] R. J. Cava, in Rare Earth Transition Metal Borocarbides (Nitrides): Superconducting, Magnetic and Normal State Properties, edited by K-H. Müller and V. N. Narozhnyi (Dordrecht: Kluwer 2001), p. 21.

[63] Y. Iwasa, K. Mizuhashi, T. Koda, Y. Tokura, G. Saito, Phys. Rev. B 49, 3580 (1994).

[64] Hiroshi Uozaki, Takahiko Sasaki, Satoshi Endo and Naoki Toyota, Journ. Phys. Soc. Jpn. 69, 2759 (2000).

[65] Claude Coulon and Rodolphe Clérac, Chem. Rev. 104, 5655 (2004).

[66] K. Miyagawa, A. Kawamoto, Y. Nakazawa, and K. Kanoda, Phys. Rev. Lett. 75, 1174 (1995).

[67] P. Wzietek, S. Lefebvre, H. Mayaffre, S. Brown, C. Bourbonnais, D. Jérome, C. Mézière and P. Batail, Hyperfine Interactions 128, 183 (2000).

[68] P. Foury-Leylekian, D. Le Bolloc'h, B. Hennion, S. Ravy, A. Moradpour, and J.-P. Pouget, Phys. Rev. B 70, 180405(R) (2004).

[69] J.-P. Pouget, P. Foury-Leylekian, D. Le Bolloc'h, B. Hennion, S. Ravy, C. Coulon, V. Cardoso, and A. Moradpour, J. Low Temp. Phys. 142, 147 (2006).

[70] Pascale Foury-Leylekian, Sylvain Petit, Claude Coulon, Bernard Hennion, Alec Moradpour and Jean-Paul Pouget, Physica B 404, 537 (2009).
[71] S. Lefebvre, P. Wzietek, S. Brown, C. Bourbonnais, D. Jérome, C. Mézière, Physica B 312-313, 578 (2002).

[72] F. Kagawa, K. Miyagawa \& K. Kanoda, Nature 436, 534 (2005).

[73] K. Kanoda, Physica C 282-287, 299 (1997), and private communication (2008).

[74] N. Biškup, L. Balicas, S. Tomić, D. Jérome, J.-M. Fabre, Phys. Rev. B 50, 12721 (1994).

[75] Xunwen Xiao, Toshiki Hayashi, Hideki Fujiwara, Toyonari Sugimoto, Satoru Noguchi, Yufeng Weng, Harukazu Yoshino, Keizo Murata, and Hiroko Aruga Katori, J. Am. Chem. Soc. 129, 12618 (2007).

[76] Wakako Suzuki, Emiko Fujiwara, Akiko Kobayashi, Yuichi Fujishiro, Eiji Nishibori, Masaki Takata, Makoto Sakata, Hideki Fujiwara, and Hayao Kobayashi, J. Am. Chem. Soc. 125, 1486 (2003).

[77] Biao Zhou, Mina Shimamura, Emiko Fujiwara, Akiko Kobayashi, Takeshi Higashi, Eiji Nishibori, Makoto Sakata, HengBo Cui, Kazuyuki Takahashi, and Hayao Kobayashi, J. Am. Chem. Soc. 128, 3872 (2006).

[78] Yohta Hara, Kazuya Miyagawa, Kazushi Kanoda, Mina Shimamura, Biao Zhou, Akiko Kobayashi, and Hayao Kobayashi, Journ. Phys. Soc. Jpn. 77, 053706 (2008).

[79] J.M. Barandiaran, D. Gignoux, D. Schmitt, J. C. GomezSal, J. Rodriguez Fernandez, P. Chieux and J. Schweizer, J. Magn. Magn. Mater. 73, 233 (1988).

[80] E. Granado, B. Uchoa, A. Malachias, R. Lora-Serrano, P. G. Pagliuso, and H. Westfahl, Jr., Phys. Rev. B 74, 214428 (2006).

[81] M. Rotter, M. Loewenhaupt, M. Doerr, A. Lindbaum, H. Sassik, K. Ziebeck, B. Beuneu, Phys. Rev. B 68, 144418 (2003).

[82] Paul M. Grant, Nature 453, 1000 (2008).

[83] Hiroki Takahashi, Kazumi Igawa, Kazunobu Arii, Yoichi Kamihara, Masahiro Hirano \& Hideo Hosono, Nature 453, 376 (2008).

[84] Yoichi Kamihara, Hidenori Hiramatsu, Masahiro Hirano, Ryuto Kawamura, Hiroshi Yanagi, Toshio Kamiya, and Hideo Hosono, J. Am. Chem. Soc. 128, 10012 (2006).

[85] E. P. Wigner, Group Theory and its Application to the Quantum Mechanics of Atomic Spectra, (Academic Press 1971).

[86] Conyers Herring, Phys. Rev. 52, 361 (1937).

[87] A. Shengelaya, H. Keller, K. A. Müller, B. I. Kochelaev, K. Conder, Phys. Rev. B 63, 144513 (2001).

[88] R. R. Ramazashvili, Jr., Zh. Eksp. Teor. Fiz. 100, 915 (1991) [Sov. Phys. JETP 73, 505 (1991)].

[89] Revaz Ramazashvili, cond-mat arXiv:0808.3946 (2008).

[90] V. V. Kabanov and A. S. Alexandrov, Phys. Rev. B 77, 132403 (2008).

[91] C. Kittel, Quantum theory of solids, (John Wiley \& Sons 1987). 